\title{
Topology design of 2D and 3D elastic material microarchitectures with crystal symmetries displaying isotropic properties close to their theoretical limits
}

\author{
R. Yera ${ }^{1}$, N. Rossi Cabral ${ }^{1}$, C.G. Méndez ${ }^{1}$, A.E. Huespe ${ }^{1,2 *}$ \\ ${ }^{1}$ CIMEC-UNL-CONICET, Predio Conicet Dr Alberto Cassano, CP 3000 Santa Fe, Argentina \\ ${ }^{2}$ E.T.S d'Enginyers de Camins, Canals i Ports, Technical University of Catalonia (Barcelona \\ Tech), Campus Nord UPC, Mòdul C-1, c/ Jordi Girona 1-3, 08034, Barcelona, Spain
}

Keywords: Three and two-dimensional (3D and 2D) microarchitecture designs; extreme isotropic elastic properties; crystal symmetries; topology optimization; inverse homogenization technique.

\begin{abstract}
This paper evaluates the effect that different imposed crystal symmetries have on the topology design of two-phase isotropic elastic composites ruled by the target of attaining extreme theoretical properties. Extreme properties are defined by the Cherkaev-Gibiansky bounds, for 2D cases, or the HashinShtrikman bounds, for 3D cases.

The topology design methodology used in this study is an inverse homogenization technique which is mathematically formulated as a topology optimization problem. The crystal symmetry is imposed on the material configuration within a predefined design domain, which is taken as the primitive cell of the underlying Bravais lattice of the crystal system studied in each case.

The influence of imposing crystal symmetries to the microstructure topologies is evaluated by testing five plane groups of the hexagonal crystal system for $2 \mathrm{D}$ problems and four space groups of the cubic crystal systems for 3D problems.

A discussion about the adequacy of the tested plane or space groups to attain elastic properties close to the theoretical bounds are presented. The extracted conclusions could be meaningful for more general classes of topology design problems in the thermal, phononic or photonic fields.
\end{abstract}

\footnotetext{
*Corresponding author. E-mail address: ahuespe@cimec.unl.edu.ar (A.E. Huespe).
} 


\section{Introduction}

The notable increase in the use of additive manufacturing techniques for processing complex material microstructures opens the possibility of realizing materials designed for given targets ([1]). In line with this problem, we present a topology design methodology for two and three-dimensional (2D and 3D) two-phase periodic microarchitectures ruled by the objective of attaining extreme isotropic elastic properties ${ }^{1}$. The crucial point in this contribution is the imposition of crystal symmetries to the designed topologies, which has to be considered as a guiding principle in the context of the adopted methodology.

The design methodology that we follow is based on a well-established mathematical tool, an inverse homogenization technique formulated as a topology optimization problem, supplemented with the necessary geometrical constraints to get the required symmetries copying that of a predefined crystal. Then, using this approach, we study the effects induced by the imposition of different space groups onto the accomplishment of the problem targets.

The study of crystal symmetries and the related mathematical method, i.e., the group theory, is one of the most fundamental topics in crystallography, particularly in crystal physics. Thus, the general concepts associated with the crystal symmetry properties, the notions of point, plane and space groups, as well as the symmetry classification which are necessary to develop this work, are taken from the International Tables for Crystallography, see [4]. Additional references are the books by Vainshtein [5] and Sólyom [6].

The space groups of 3D periodic arrangements of crystals characterize their symmetry properties. Symmetry elements of these space groups are constituted by rotation axes, mirror-reflection planes, inversion-rotation axes, glide-reflection planes, and screw axes. The point groups are the nexus, via the Neuman's theorem, between the symmetry of the microstructure topology, defined by the crystal space group, and the symmetry of the macroscopic effective physical properties, see Nye [7]. This concept is the cornerstone of the present approach.

Inverse homogenization techniques for topology designs are well-established in the literature. In this sense, the work pioneered by Sigmund [8], the numerous posterior contributions of this author and coworkers, as well as the huge number of papers addressed to this topic (see for example Torquato [9] and Osanov and Guest [10]), are a clear indication of the power of this technique to obtain new microarchitecture topologies which are hard to be imagined if using ad-hoc design

\footnotetext{
${ }^{1}$ The concept of extreme material has been coined by Milton and Cherkaev [2]. They give this name to naterials which elasticity tensors have some eigenvalues very large and the remaining ones are very small. Here, following to Sigmund [3], we use this concept in a slightly broader sense, including all those isotropic composites whose effective properties attain extreme bulk and shear modulus.
} 
methodologies, see, in particular, the brief discussion by Milton [11]. Inverse homogenization techniques can be formulated as a mathematical topology optimization problem posed in a spatial domain $\Omega_{\mu}$. Their solutions provide the optimal topologies, or spatial material layouts, satisfying the proposed targets. An overview of these techniques for different problems can be found in the book by Bendsoe and Sigmund [12].

We closely follow the works of Sigmund [3] and Andreassen and Lazarov [13], where, 3D (2D) microarchitecture design problems aiming at obtaining extreme composites using inverse homogenization techniques have been reported. Here, we solve the topology optimization algorithm with the necessary geometrical constraints to impose a space (plane) group to the obtained material layout. The space (plane) group is defined apriori according to the symmetry of the target effective elastic response.

The implementation of this approach has required the development of a particular numerical treatment for handling the high computational cost demanded by 3D microstructure designs. Nevertheless, we leave for a forthcoming paper a detailed description of the numerical aspects of the methodology. It is notably to remark here that the topology optimization algorithm is solved with a spatial filter to avoid the occurrence of thin microstructures. Therefore, the attained topologies have only one length scale. The authors have previously reported some studies performed with this methodology for 2D problems in Podestá et al. [14] and Podestá et al. [15] and 3D problems in Méndez et al. [16].

In this paper, initially and after a summary of the concepts on which this work is based on, we evaluate in Section 3 several plane groups to design 2D microarchitectures aiming at obtaining extreme elastic properties. The material isotropy is guaranteed by taking plane groups of the hexagonal crystal system. Subsequently, in Section 4, we perform a similar analysis for 3D problems. In these cases, the isotropic elastic response cannot be guaranteed by any space group ${ }^{2}$. Thus, we test four space groups with the highest and the lowest symmetries of the cubic crystal system, adding one isotropy constraint to the topology optimization problem. The 2D and 3D material configurations obtained with different plane and space groups are compared and discussed. Finally, the conclusions are presented.

Some representative 3D microstructures designed with this methodology are available as supplementary material in the dataset Yera et al. [17]. These microarchitectures are stored in .stl format and are ready for 3D printing.

\footnotetext{
${ }^{2}$ It is worth to remark that, when topologies with multiple length scales are allowed, the crystal symmetry does not matter for attaining isotropic effective responses, such as happens in randomly textured polycrystals with isotropic macroscopic response. However, the important point here is that topologies implying multiple length scales are not so practical and their consideration enlarges the design space which makes the optimization more difficult.
} 


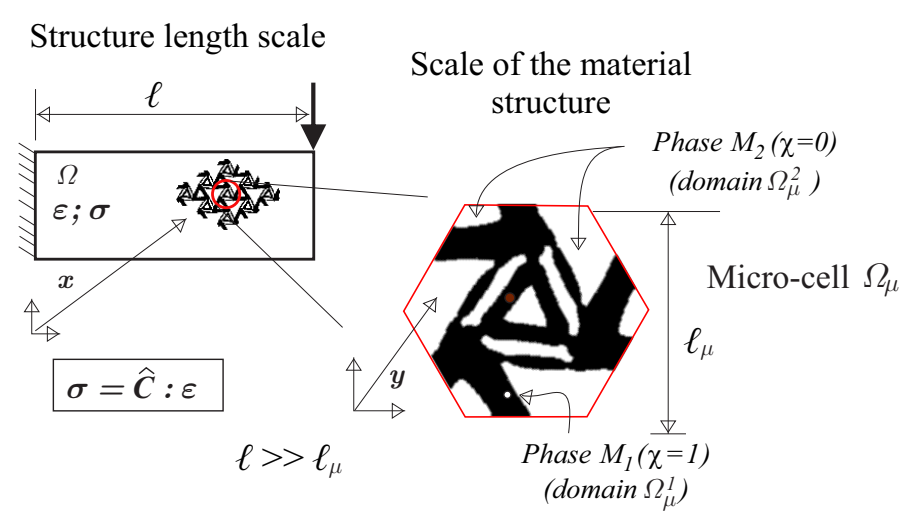

Figure 1: Topology optimization problem. Cell $\Omega_{\mu}$ of the microstructured composite with phases $M_{1}$ and $M_{2}$ and the characteristic function $\chi$. At the macrostructural scale, the effective elastic properties of the composite are represented by the homogenized constitutive elasticity tensor $\hat{\boldsymbol{C}}$.

\section{Inverse material design problem}

We design microstructures of periodic composites constituted by two isotropic phases, $M_{1}$ and $M_{2}$, and a given volume fraction $f_{1}$ of $M_{1}$. Two well-separated scales of lengths are assumed, see Figure 1 ; the structure length scale, $\ell$, and the microstructural length scale $\ell_{\mu}$ in where the geometrical configuration of the phase distribution is defined. Thus, it is satisfied that $\ell \gg \ell_{\mu}$. The effective material properties are evaluated at the structural scale.

The composite has to display the closest effective isotropic elastic properties to its theoretically estimated bounds. In particular, for the here studied cases in 2D problems, these bound have been reported by Cherkaev and Gibiansky [18], and for 3D problems, they have been reported by Hashin and Shtrikman [19]. The elastic properties of the component phases are chosen to allow for effective properties with negative PoissonâẮs ratios. Thus, the design of isotropic auxetic materials is an additional challenge addressed for some extreme target conditions.

In this Section, and after defining the theoretical bounds reported in the literature, we describe the optimization problems that are formulated to attain the closest properties to these bounds. 


\subsection{Analytical bounds of two-phase composites with effective isotropic elasticity}

The bounds described in this Section for the effective bulk and shear moduli, $\hat{\kappa}$ and $\hat{G}$, respectively ${ }^{3}$, of isotropic two-phase composites, are valid for well-ordered composites, i.e. $\kappa_{2}<\kappa_{1}$ and $G_{2}<G_{1}$, where $\kappa_{1}$ and $G_{1}$ are the bulk and shear moduli of the stiff phase, $M_{1}$, and $\kappa_{2}$ and $G_{2}$ are the bulk and shear moduli of the soft phase, $M_{2}$. These bounds are next defined in the space $(\hat{K}, \hat{G})$ for 2 D problems ${ }^{4}$ and $(\hat{\kappa}, \hat{G})$ for $3 \mathrm{D}$ problems.

Plane strain problems. According to the analysis of Cherkaev et al., the effective moduli of an isotropic composite constituted by the phase $M_{1}$, with bulk modulus ${ }^{5}$ $K_{1}=5 / 7$, shear modulus $G_{1}=5 / 13$ and volume fraction $f_{1}=0.5$, and the phase $M_{2}$, with bulk modulus $K_{2}=K_{1} / 200$ and shear modulus $G_{2}=G_{1} / 200$; can be bounded in the space $(\hat{K}, \hat{G})$ with an upper coupled bound defined by the curve:

$$
\hat{G}^{u}(\hat{K})=\frac{\omega^{u}\left(f_{1} G_{1}+f_{2} G_{2}\right)+G_{1} G_{2}}{G_{2} f_{1}+G_{1} f_{2}+\omega^{u}} ; \quad \omega^{u}=-G_{1}+\frac{\alpha^{u} y_{K}}{\beta^{u} y_{K}-\gamma^{u}} ;
$$

and a lower coupled bound, defined by the curve:

$$
\hat{G}^{l}(\hat{K})=\frac{\omega^{l}\left(f_{1} G_{1}+f_{2} G_{2}\right)+G_{1} G_{2}}{f_{1} G_{2}+f_{2} G_{1}+\omega^{l}} \quad ; \quad \omega^{l}=-G_{1}+\frac{\alpha^{l}\left(\frac{1}{y_{K}}+\frac{1}{K_{1}}\right)}{\beta^{l}\left(\frac{1}{y_{K}}+\frac{1}{K_{1}}\right)-\gamma^{l}} ;
$$

where

$$
y_{K}=-K_{1} K_{2} \frac{\hat{K}-\frac{1}{\left(\frac{f_{1}}{K_{1}}+\frac{f_{2}}{K_{2}}\right)}}{\hat{K}-\left(f_{1} K_{1}+f_{2} K_{2}\right)}\left(\frac{f_{1}}{K_{1}}+\frac{f_{2}}{K_{2}}\right)
$$

The coefficient in (1)-(2) are: $\alpha^{l}=0.4107, \beta^{l}=1.0653, \gamma^{l}=0.0110, \alpha^{u}=-0.1256$, $\beta^{u}=-0.2200$ and $\gamma^{u}=2.0 \times 10^{-4}$.

The curves $\left(\hat{K}, \hat{G}^{u}\right)$ and $\left(\hat{K}, \hat{G}^{l}\right)$ are plotted in Figure 2 -a and, in the following, are denoted CG-bounds.

Three-dimensional problems. The best known bounds at the present time for 3D composites, when one phase is void, are the Hashin-Shtrikman bounds (denoted

\footnotetext{
${ }^{3}$ The symbol $\left({ }^{\wedge}\right)$ denotes an effective value of the elastic property.

${ }^{4}$ The plane strain modulus $K$ is given by $K=\kappa+G / 3$, where $\kappa$ and $G$ are the conventional bulk and shear moduli of the three-dimensional theory. The elastic constant in the plane strain $\nu_{p}=\nu /(1-\nu)$, where $\nu$ is the conventional 3D Poisson's ratio and $-1 \leq \nu_{p} \leq 1$, is here taken as the plane strain Poisson's ratio. The parameter $K$ is known as the Kolosov constant. For additional details about the connection between the $2 \mathrm{D}$ and $3 \mathrm{D}$ elastic constants we reference the work by Thorpe and Jasiuk [20].

${ }^{5}$ Stifnesses and lengths are defined in arbitrary units.
} 

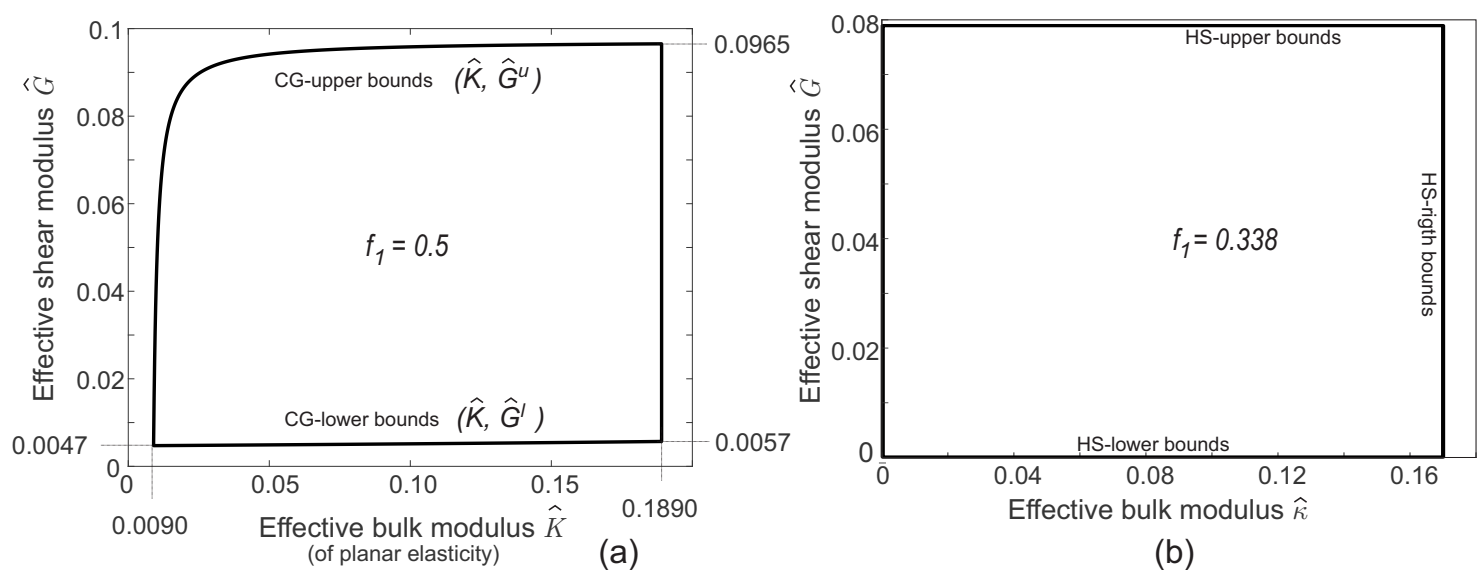

(b)

Figure 2: Analytic bounds of effective properties for two-phase isotropic composites. a) Plane elasticity. Bounds according to Cherkaev and Gibiansky [18] (CG-bounds). Properties of phases $M_{1}$ and $M_{2}$ are $K_{1}=5 / 7 ; K_{2}=K_{1} / 200 ; G_{1}=5 / 13 ; G_{2}=$ $G_{1} / 200$. Volume fraction of phase $M_{1}$ is $f_{1}=0.5$. b) $3 \mathrm{D}$ elasticity bounds according to Hashin and Shtrikman [19] (HS-bounds). Properties of phases $M_{1}$ and $M_{2}$ are $\kappa_{1}=1.667, G_{1}=0.3571, \kappa_{2}=\gamma \kappa_{1}, G_{2}=\gamma G_{1}$, contrast factor $\gamma=10^{-4}$, volume fraction $f_{1}=0.338$.

HS-bounds in the following) which are given by the expressions:

$$
\begin{gathered}
\hat{\kappa}^{u}=\kappa_{1}+\frac{1-f_{1}}{\frac{1}{\kappa_{2}-\kappa_{1}}+3 \frac{f_{1}}{3 \kappa_{1}+4 G_{1}}} \quad ; \quad \hat{\kappa}^{l}=\kappa_{2}+\frac{f_{1}}{\frac{1}{\kappa_{1}-\kappa_{2}}+3 \frac{1-f_{1}}{3 \kappa_{2}+4 G_{2}}} \\
\hat{G}^{u}=G_{1}+\frac{1-f_{1}}{\frac{1}{G_{2}-G_{1}}+\frac{6\left(\kappa_{1}+2 G_{1}\right) f_{1}}{5 G_{1}\left(3 \kappa_{1}+4 G_{1}\right)}} \quad ; \quad \hat{G}^{l}=G_{2}+\frac{f_{1}}{\frac{1}{G_{1}-G_{2}}+\frac{6\left(\kappa_{2}+2 G_{2}\right)\left(1-f_{1}\right)}{5 G_{2}\left(3 \kappa_{2}+4 G_{2}\right)}} .
\end{gathered}
$$

By following to Andreassen and Lazarov [13], we adopt $\kappa_{1}=1.667, G_{1}=0.3571$, $\kappa_{2}=\gamma \kappa_{1}, G_{2}=\gamma G_{1}$, with the contrast factor $\gamma=10^{-4}$, and the volume fraction $f_{1}=0.338$. For these composites, the HS-bounds are: $\hat{\kappa}^{u}=0.170 ; \hat{\kappa}^{l}=2.8 \times 10^{-4}$; $\hat{G}^{u}=0.789 ; \hat{G}^{l}=7.3 \times 10^{-5}$ and are plotted in Figure 2 -b, in the space $(\hat{\kappa}, \hat{G})$.

Remark: in two-dimensions the Cherkaev-Gibiansky bounds degenerate to the Hashin-Shtrikman bounds in the limit in which one phase is void. This is similar to the way the Berryman-Milton-Phan-Thien bounds degenerate in three dimensions to the Hashin-Shtrikman bounds, see Berryman and Milton [21] and references cited therein. 


\subsection{Topology optimization problem}

Let us consider a basic micro-cell, identified by $\Omega_{\mu}$, of the two-phase composite. The phases $M_{1}$ and $M_{2}$ occupy the domains $\Omega_{\mu}^{1}$ and $\Omega_{\mu}^{2}$, respectively, see Figure 1 .

In $\Omega_{\mu}$, we define a characteristic function $\chi(\boldsymbol{y})$ identifying the positions where the phase $M_{1}$ is placed. It is defined by:

$$
\chi(\boldsymbol{y})=\left\{\begin{array}{ll}
0 & \forall \boldsymbol{y} \in \Omega_{\mu}^{2} \\
1 & \forall \boldsymbol{y} \in \Omega_{\mu}^{1}
\end{array} .\right.
$$

The homogenized elasticity tensor of the composite, $\hat{\boldsymbol{C}}$, depends on the geometrical configuration of the phases $M_{1}$ and $M_{2}$ in $\Omega_{\mu}$. We make explicit this dependence by introducing the notation $\hat{\boldsymbol{C}}(\chi)$. If the effective properties of the composite are isotropic, then, $\hat{\boldsymbol{C}}$ can be determined accordingly with the effective bulk and shear moduli, denoted $\hat{\kappa}(\chi)$ and $\hat{G}(\chi)$, respectively.

\subsubsection{Three-dimensional topology optimization problems}

The optimal design of microarchitecture topologies whose target effective properties are the points on the lower Hashin-Shtrikman bounds are obtained by solving a set of $n$ discrete topology optimization problems formulated as follows:

$$
\begin{array}{cc}
\min _{\chi} \hat{G}(\chi) ; \\
\text { such that: } \quad \hat{\kappa}(\chi)-\kappa_{j}^{*}=0 \\
& \hat{\boldsymbol{C}}(\chi) \text { is isotropic } \\
& f_{1}(\chi)-f_{1}^{*}=0
\end{array}
$$

where $\kappa_{j}^{*}$ (with $1 \leq j \leq n$ ) is the $j$-th target bulk modulus of the composite defined whitin the interval $\hat{\kappa}^{l} \leq \kappa_{j}^{*} \leq \hat{\kappa}^{u}$ and $f_{1}^{*}$ is the target volume fraction of phase $M_{1}$. The problem (6) expresses that the minimum of the effective shear modulus, $\hat{G}$, is searched by varying the characteristic function $\chi$ within $\Omega_{\mu}$.

Similarly, the Hashin-Strikman upper bound is approached by solving the maximum $\hat{G}(\chi)$, with identical constraints of problem (6).

Alternatively, the topologies approaching the left Hashin-Strikman bound are sought by solving the problems:

$$
\min _{\chi} \hat{\kappa}(\chi)
$$

$$
\begin{array}{ll}
\text { such that: } \quad & \hat{G}(\chi)-G_{j}^{*}=0 \\
& \hat{\boldsymbol{C}}(\chi) \text { is isotropic } \\
& f_{1}(\chi)-f_{1}^{*}=0
\end{array}
$$


where now, the target effective shear modulus $G_{j}^{*}$ of the $j$-th optimization problem is chosen from the the interval $\hat{G}^{l} \leq G_{j}^{*} \leq \hat{G}^{u}$. The extreme composites on the right $\mathrm{H}-\mathrm{S}$ bound are approached by changing the minimum problem in (7) by one of maximizing the effective bulk modulus.

In the case that the effective elastic properties have cubic symmetry, situation that is guaranteed by taking a material configuration having a space group consistent with the cubic crystal system, the coefficients $\hat{C}_{i j}$, with $i, j=1, \ldots, 6$, of the elasticity matrix $^{6} \hat{\boldsymbol{C}}$ satisfy the general identities displayed in Table 2 , resulting: $\hat{C}_{11}=\hat{C}_{22}=$ $\hat{C}_{33} ; \hat{C}_{12}=\hat{C}_{13}=\hat{C}_{23} ; \hat{C}_{44}=\hat{C}_{55}=\hat{C}_{66}$. The remaining coefficients of the elasticity matrix are zero. Any tensor $\hat{\boldsymbol{C}}$ with cubic symmetry is isotropic if additionally its coefficients satisfies:

$$
\hat{C}_{11}-\hat{C}_{12}-\hat{C}_{44}=0
$$

resulting:

$$
\begin{aligned}
\hat{G}^{i s o} & =\frac{\hat{C}_{44}}{2}, \\
\hat{\kappa}^{i s o} & =\frac{1}{3}\left(\hat{C}_{11}+2 \hat{C}_{12}\right),
\end{aligned}
$$

where $\hat{\kappa}^{i s o}$ and $\hat{G}^{i s o}$ are the bulk and shear moduli of the effective elastic isotropic response. In the problems (6) and (7), the elastic isotropy is imposed by the equation (8).

Based on these identities we reformulate the problem (6) in terms of the components of $\hat{\boldsymbol{C}}$ as follows:

$$
\begin{array}{cc}
\min _{\chi} \hat{C}_{44}, \\
\text { such that: } \quad \hat{C}_{11}+2 \hat{C}_{12}-3 \kappa_{j}^{*}=0, \\
& \hat{C}_{11}-\hat{C}_{12}-\hat{C}_{44}=0, \\
& f_{1}(\chi)-f_{1}^{*}=0,
\end{array}
$$

and problem (7) as follows:

$$
\begin{aligned}
\min _{\chi}\left(\hat{C}_{12}+\frac{1}{3} \hat{C}_{44}\right) & \\
\text { such that: } \quad & \left(\hat{C}_{11}-\hat{C}_{12}+\frac{3}{2} \hat{C}_{44}\right)-5 G_{j}^{*}=0, \\
& \hat{C}_{11}-\hat{C}_{12}-\hat{C}_{44}=0 \\
& f_{1}(\chi)-f_{1}^{*}=0 .
\end{aligned}
$$

The objective function in problem (12) results from replacing the isotropy constraint (8) in equation (10).

\footnotetext{
${ }^{6}$ We use Kelvin's notation.
} 


\subsubsection{Two-dimensional (plane strain) topology optimization problems}

The optimal design of 2D microstructures whose target is to attain an extreme isotropic material is also performed using a topology optimization algorithm with a slightly different formulation to those stated in (6) or (7). In this case, those problems are formulated without specifically imposing the isotropic elasticity constraint, because this property is guaranteed by enforcing topologies with hexagonal symmetry.

\subsubsection{Topology optimization algorithm}

The algorithm used for solving the problems (11)-(12) is based on a level-set method jointly with topological derivative evaluating the sensitivity of response with changes of the characteristic function $\chi$ defined in (5). The numerical technique follows the original proposal of Amstutz and Andrä [22] and Amstutz et al. [23]. The topological derivative has been studied by Novotny and Sokołowski [24]. Additional details of this algorithm for solving 3D problems can be found in Méndez et al. [16].

In all cases, the configurations for the algorithm onset are random distributions of the stiff phase in $\Omega_{\mu}$.

\subsection{Use of specific plane group and space group symmetries}

We focus only on particular cases of space group symmetries for designing composites with isotropic effective elastic responses.

\subsubsection{Hexagonal crystal system for 2D isotropic material design}

The isotropy of 2D elastic properties is guaranteed if the periodic material configuration is compatible with the hexagonal crystal system whose main properties are summarized in Table 1 . Thus, the plane groups $p 3, p 3 m 1, p 31 m, p 6$, and $p 6 m m^{7}$ guarantee the obtention of isotropic effective elastic properties, and therefore, they are the ones tested in this work.

The symmetry elements of these plane groups are depicted in Figure 3. The underlying Bravais lattice compatible with these plane groups is hexagonal.

\footnotetext{
${ }^{7}$ We use the Herrman-Maugin notation to identify point, plane and space groups, see International Tables of Crystallography [4].
} 
Table 1: Hexagonal (2D) Crystal System. Compatible point and plane groups. The elasticity matrix is isotropic and is represented in column 1, with the coefficcient $C_{11}$ and $C_{12}$ characterizing the elastic properties.

\begin{tabular}{|c|c|c|c|c|c|c|c|}
\hline \multicolumn{3}{|c|}{$\begin{array}{l}\text { Elasticity } \\
\text { Matrix }\end{array}$} & $\begin{array}{l}\text { Crystal } \\
\text { system }\end{array}$ & $\begin{array}{l}\text { Point } \\
\text { group }\end{array}$ & $\begin{array}{l}\text { Plane } \\
\text { group }\end{array}$ & $\begin{array}{c}\text { Compatible } \\
\text { Bravais lattice }\end{array}$ & $\begin{array}{l}\text { Wigner-Seitz and } \\
\text { primitive unit cell }\end{array}$ \\
\hline \multirow{5}{*}{$\begin{array}{c}C_{11} \\
C_{12} \\
0\end{array}$} & \multirow{5}{*}{$\begin{array}{c}C_{12} \\
C_{11} \\
0\end{array}$} & \multirow{5}{*}{$\left.\begin{array}{c}0 \\
0 \\
C_{11}-C_{12}\end{array}\right]$} & \multirow{5}{*}{ hexagonal } & 3 & p3 & \multirow{5}{*}{ hexagonal } & \multirow{5}{*}{ • } \\
\hline & & & & \multirow{2}{*}{$3 \mathrm{~m}$} & p3m1 & & \\
\hline & & & & & p31m & & \\
\hline & & & & 6 & p6 & & \\
\hline & & & & $6 \mathrm{~mm}$ & p6mm & & \\
\hline
\end{tabular}
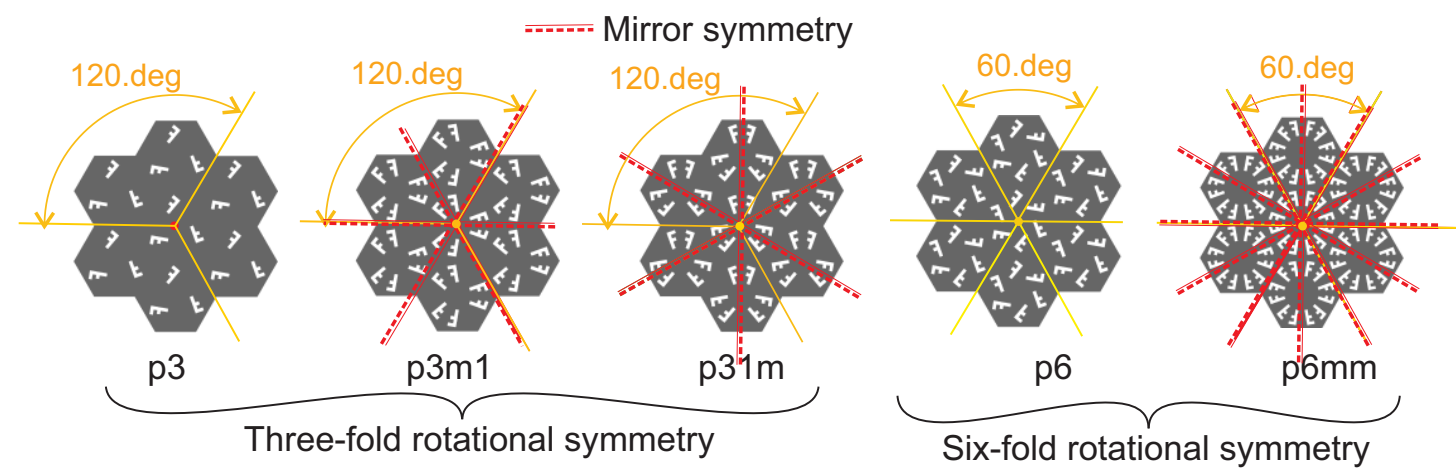

Figure 3: Symmetry elements of plane groups $p 3, p 3 m 1, p 31 m, p 6, p 6 m m$. Note that the mirror planes of the $p 3 m 1$ plane group intersect the Voronoi cell differently to that of the $p 31 m$ plane group. 
Table 2: Cubic System. Compatible point and space groups. Two Bravais lattices compatible with the cubic systems are SC and BCC. Conventional (or Centered) and Wigner-Seitz cells of the two Bravais lattices. The elasticity matrix in the natural bais is represented in column 1 . The symbol "*-*" linking two coefficients means that they are equal. Thus, only three coefficients define the elasticity matrix in natural basis. The evaluated point and space groups are denoted in bold.

\begin{tabular}{|c|c|c|c|c|}
\hline $\begin{array}{l}\text { Elasticity } \\
\text { Tensor }\end{array}$ & $\begin{array}{l}\text { Crystal } \\
\text { System }\end{array}$ & $\begin{array}{l}\text { Point } \\
\text { Groups }\end{array}$ & \multicolumn{2}{|c|}{$\begin{array}{l}\text { Space } \\
\text { Groups }\end{array}$} \\
\hline \multirow{5}{*}{ 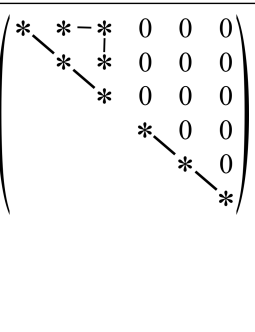 } & \multirow{5}{*}{ Cubic } & 23 & P23, $\mathrm{P} 2{ }_{1} 3$ & $\mathbf{I} 23, I 2_{1} 3$ \\
\hline & & $\mathrm{m} 3$ & $\mathrm{Pm} 3, \mathrm{Pn} 3, \mathrm{~Pa} 3$ & $\operatorname{Im} 3, \operatorname{Ia} 3$ \\
\hline & & 432 & $\begin{array}{l}\mathrm{P} 432, \mathrm{P} 4_{2} 32 \\
\mathrm{P} 4_{3} 32, \mathrm{P} 4_{1} 32\end{array}$ & $\mathrm{I} 432, \mathrm{I} 4_{1} 32$ \\
\hline & & $\overline{43 m}$ & $\mathrm{P} \overline{4} 3 \mathrm{~m}, \mathrm{P} \overline{4} 3 \mathrm{n}$ & $\mathrm{I} \overline{4} 3 \mathrm{~m}, \mathrm{I} \overline{4} 3 \mathrm{~d}$ \\
\hline & & $\mathbf{m} \overline{\mathbf{3}} \mathbf{m}$ & $\begin{array}{c}\mathbf{P m} \overline{3} \mathbf{m}, \operatorname{Pn} \overline{3} n, \\
\operatorname{Pm} \overline{3} n, \operatorname{Pn} \overline{3} m\end{array}$ & $\operatorname{Im} \overline{\mathbf{3}} \mathbf{m}$, Ia $\overline{3} \mathrm{~d}$ \\
\hline \multicolumn{3}{|c|}{$\begin{array}{c}\text { Compatible Bravais lattice } \\
\text { (Conventional cells) }\end{array}$} & $\mathrm{SC}$ & $\mathrm{BCC}$ \\
\hline Wigner & Seitz ce & & $\mathrm{sCl}$ & $\mathrm{BCC}$ \\
\hline
\end{tabular}

\subsubsection{Cubic crystal system for 3D isotropic material design}

There are 230 space groups characterizing periodic microstructures in 3D. None of them guarantee an isotropic effective elastic response. Under this circumstance, we study the cubic space groups, which have the highest possible crystal symmetries in 3D. We only focus on the cubic crystal system whose Bravais lattices are SC (primitive Simple Cubic) or BCC (Body-Centered Cubic). A further analysis would include the FCC lattice.

Additional details about the cubic crystal system are presented in Table 2. Column 4 of this Table displays all the space groups that are compatible with the SC and BCC lattices. Point and space groups being compatible are described in identical lines of columns 3 and 4 . It can be guaranteed that the effective elasticity properties of any composite whose material layout has a symmetry compatible with a point group listed in column 3 satisfy cubic symmetry, and therefore, the corresponding elastic matrix coefficients, expressed in natural basis, should satisfy the identities shown in column 1 . In the last two rows of Table 2, we show the Conventional and 
Wigner-Seitz (Voronoi) cells of both lattices which are here studied ${ }^{8}$. Primitive cells of this lattice are shown in Figure 4. The primitive and the conventional unit cells are identical for the $\mathrm{SC}$ lattice and different for the $\mathrm{BCC}$ lattice.
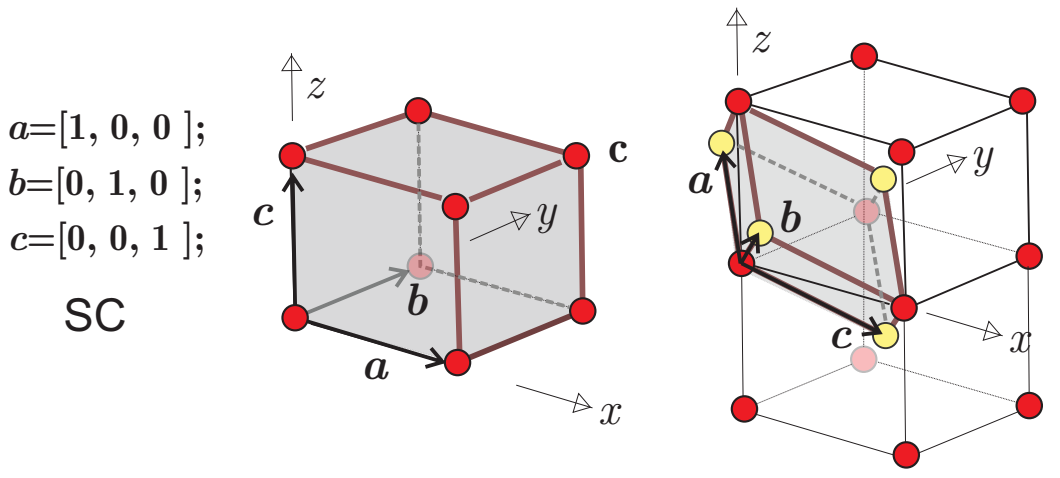
$a=\left[\begin{array}{lll}-0.5, & 0.5 & 0.5\end{array}\right]$

$b=\left[\begin{array}{ll}0.5,-0.5 & 0.5\end{array}\right]$

$c=\left[\begin{array}{lll}0.5, & 0.5 & -0.5\end{array}\right]$

$\mathrm{BCC}$

Figure 4: Primitive cells of cubic Bravais Lattices of SC and BCC lattices are defined by the primitive vectors $\boldsymbol{a}, \boldsymbol{b}, \boldsymbol{c}$. The volumes of the primitive unit cells are $V_{S C}=1, V_{B C C}=0.5$. Conventional cells are defined in both cases by the vectors $(1,0,0),(0,1,0)$ and $(0,0,1)$.

We only analyze the design of topologies with space groups $P 23, I 23, P m \overline{3} m$ and $\operatorname{Im} \overline{3} \mathrm{~m}$. Some of the symmetry elements of these space groups are shown in Figure 5 .

The space groups $P 23$ and $I 23$ have fewer symmetry elements than the $P m \overline{3} m$ and $\operatorname{Im} \overline{3} m$ cases. The first ones can be considered as the space groups with the lowest symmetries and the second ones with the highest of the cubic crystal system. Also, the space groups P23 and Pm3m are compatible with an SC Bravais lattice, while the $I 23$ and $I m \overline{3} m$ are compatible with a BCC Bravais lattice. Note further that the space group $I 23$ has two-fold screw axes which provide higher symmetry properties than that of the space group P23. As will be observed in the following, this higher symmetry of the $I 23$ space group affects the capacity for obtaining some minimal/maximal properties close to the extreme materials.

The objective here is to make a comparative analysis of the topologies obtained using both extreme cases of symmetries, with point groups 23 and $m \overline{3} m$, and two different Bravais lattices, SC and BCC.

\subsection{Technique for solving the inverse design problem}

Significant issues for solving the topology optimization algorithm summarized in sub-Section 2.2.3 are next remarked.

\footnotetext{
${ }^{8}$ In this work, three types of unit cells are mentioned and used: i) Wigner-Seitz (or Voronoi) unit cells; ii) primitive unit cells defined by the primitive vectors of the lattice and iii) the conventional cells whose faces are planes parallel to the coordinate planes. Voronoi and primitive cells have minimum volume, while conventional cells, in general, do not have minimum volume
} 

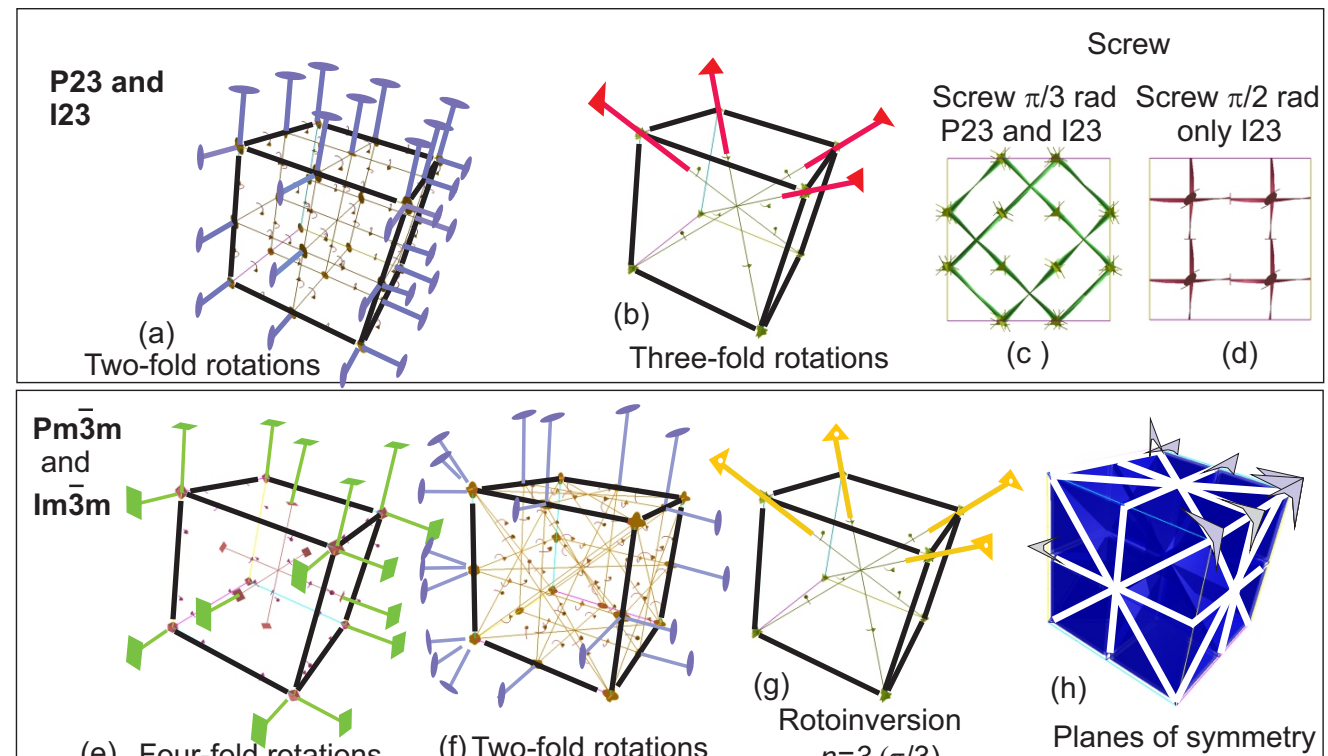

$\begin{array}{ll}\text { (e) Four-fold rotations } & \text { (f) Two-fold rotations }\end{array}$
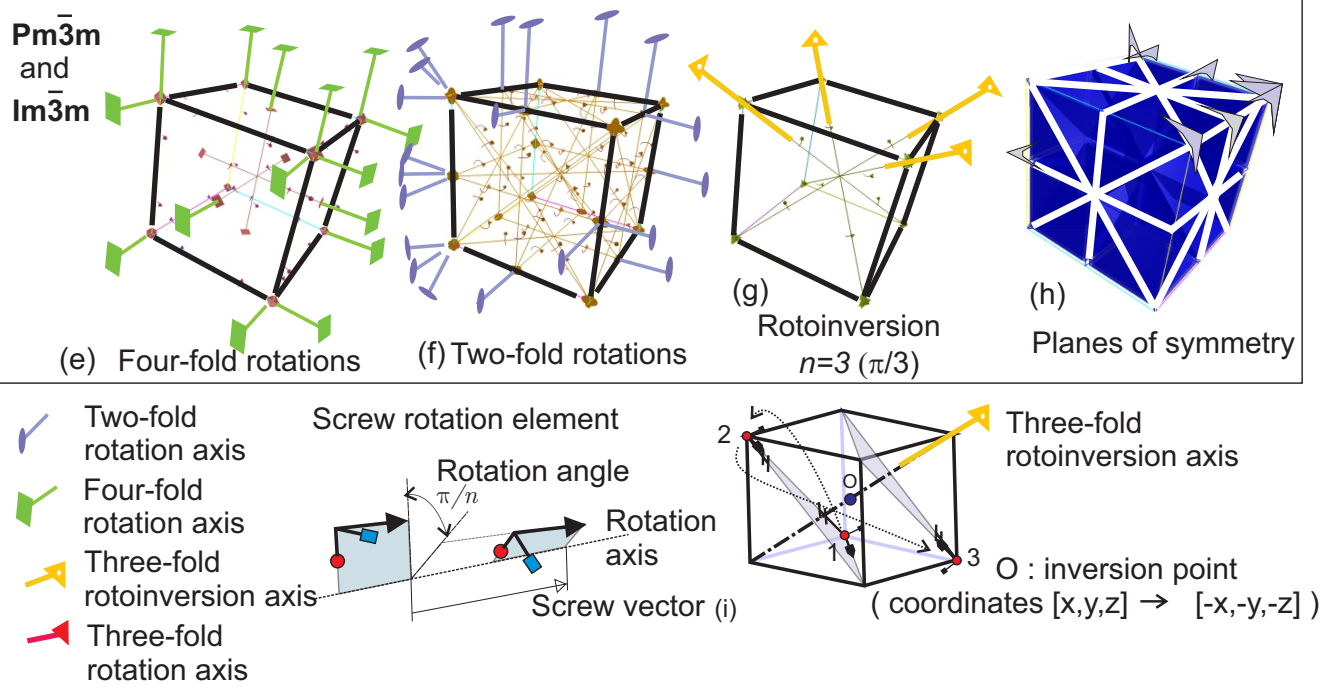

Figure 5: (a) to (h) Symmetry elements in conventional SC and BCC cells, of the space groups $P 23, I 23, P m \overline{3} m$ and $I m \overline{3} m 3$. Glide and screw elements of space groups $\operatorname{P} m \overline{3} m$ and $\operatorname{Im} \overline{3} m 3$ are not shown. c) and d) orthogarphic view of screw elements of space groups $P 23$ and 123 . i) representation of the screw rotation and rotoinversion symmetry elements. The symmetry elements of space groups can be interactively visualized with the software "Space Group Visualizer" described in [25]. 
i) Selection of the design domain $\Omega_{\mu}$ : following to Podestá et al. [15], $\Omega_{\mu}$ is taken as the primitive cell of a Bravais lattice having a point group symmetry compatible with the target elastic properties. This lattice is identified as the underlying Bravais lattice of the composite. For 2D problems, the hexagonal Bravais lattice is taken, and the corresponding primitive cell is the parallelogram depicted in Figure 6. In 3D problems, the domain $\Omega_{\mu}$ coincides with the primitive cell of the underlying Bravais lattice ${ }^{9}$. The primitive cells of SP and BCC lattices are shown in Figure 4.

ii) Computational homogenization: the effective properties of the composite is evaluated using a base cell coinciding with $\Omega_{\mu}$. Periodic conditions of the material layout, by repeating the pattern defined by this base cell, are coincident with the primitive directions of the underlying Bravais lattice. Thus, periodic boundary conditions of the displacement fluctuations compatible with this criterion are imposed on the base cell.

In $3 \mathrm{D}$ problems, the homogenization of the effective properties is computed with an FFT (Fast Fourier Transform) technique ${ }^{10}$.

iii) Implementation of the space group symmetry: the symmetry of the microarchitecture topology agreeing with a pre-established space group is imposed in two steps. The first step consists of defining a set of points in the primitive cell, known as the asymmetric unit domain ${ }^{11}$, and then to find their image points. The asymmetric domain is the reduced volume of the primitive cell which can be replicated by applying the symmetry operations of the space group to obtain the full primitive cell configuration. Therefore, the asymmetric unit contains the complete information necessary for the description of the crystal structure. In Figure 6, we depict the plane group consistent with the hexagonal crystal system and the asymmetric unit domains in red. In different colors are depicted the image points. The asymmetric unit domains, as well as the image points of the conventional cells for all plane and space groups, are defined in the International Tables for Crystallography ([4]).

\footnotetext{
${ }^{9}$ Selecting the primitive unit cell of the Bravais lattice instead of the conventional cell means that the design domain would represent the minimum possible volume of the composite which tesselation reproduce the periodic microstructure. Then, for identical microstructures, the minimum volume of $\Omega_{\mu}$, instead of using a conventional cell, implies to search for a simpler material configuration.

${ }^{10}$ The FFT technique for homogenization implemented in this work has been taken from Eyre and Milton [26]. In 2D, this step of the inverse homogenization problem is computed with a Finite Element technique. Additional details of the numerical aspects of this technique are addressed in Rossi Cabral et al. [27].

${ }^{11}$ See point 2.1.3.8 in[4]
} 


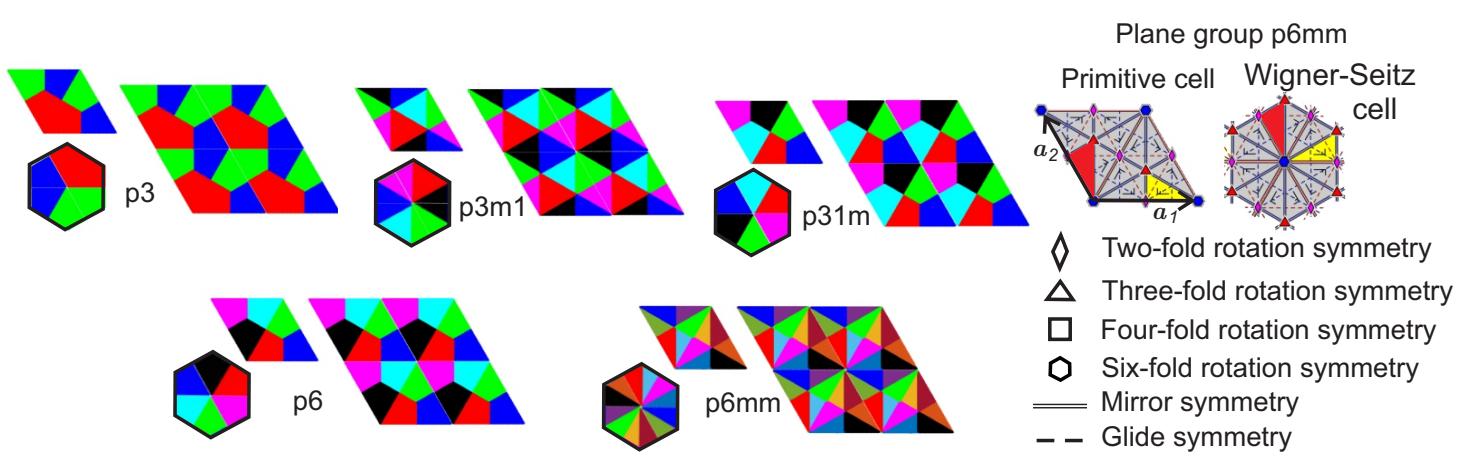

Figure 6: Five plane groups of the hexagonal crystal system. Asymmetric units in red and their image points. The asymmetric unit domain is easily distinguished in the Wigner-Seitz cell but hardly recognized in primitive cells. Symbols used for identifying the symmetry elements in plane groups.

\section{Design of 2-D isotropic materials}

The attainment of a 2D microstructure with isotropic effective elastic response is strictly guaranteed ${ }^{12}$ by imposing the following plane groups $p 3, p 3 m 1, p 31 m, p 6$ and $p 6 \mathrm{~mm}$ which are ordered from the lowest to the highest symmetries. We take a primitive unit cell of the hexagonal Bravais lattice to solve the topology optimization algorithm.

The designs of materials whose properties are close to the bounds, for two-phase composites defined in Section 2.1, are studied for 13 different target conditions identified by the corresponding points in the space $(\hat{K}, \hat{G})$ depicted in Figure 7 -a. The bounds reported by Cherkaev et al. are displayed again in the Figure, as well as some isolines of negative Poisson's ratios. The Point NP marks the elastic properties with the most negative Poisson's ratio estimated with the CG-bounds.

\subsection{Discussion of results}

After evaluating these solutions, the following discussion can be addressed.

1) The enforcement of a plane group with the highest symmetry $(p 6 m m)$ is the most adequate to attain microarchitectures with elastic properties close to the region with low shear and high bulk moduli, i.e., near the Walpole Point (Point WP in Figure 7-a and c);

2) The enforcement of a plane groups with a hexagonal Bravais lattice and only few symmetry elements guaranteeing isotropy $(p 3, p 31 m$ and $p 6)$ are useful to

\footnotetext{
${ }^{12}$ The effective elasticity tensor of all the 2D microarchitectures displayed in this Section are exactly isotropic until the machine precision.
} 


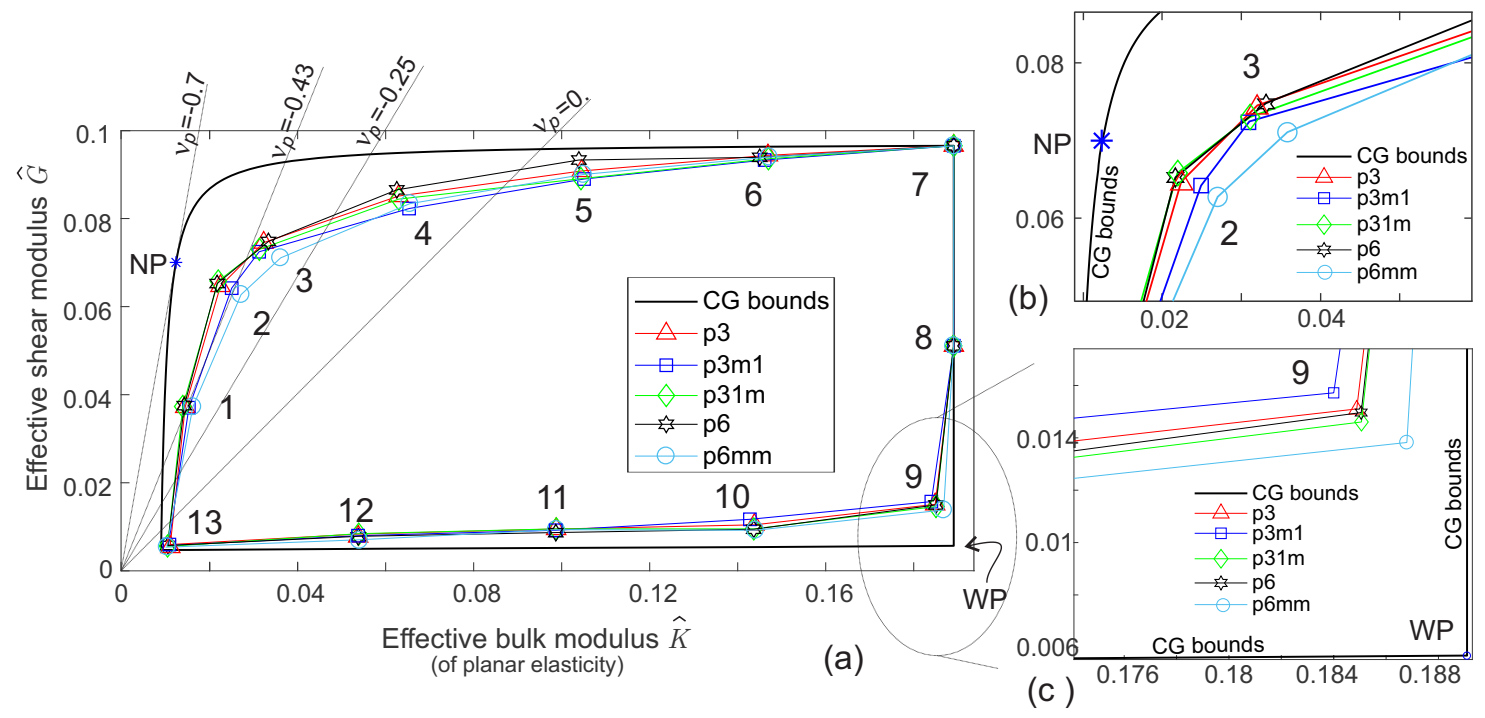

Figure 7: Two-dimensional design of extreme isotropic microstructures: a) Cherkaev and Gibiansky [18] bounds (CG-bounds)) and best solutions obtained with plane groups $p 3, p 3 m 1, p 31 m, p 6$ and $p 6 m m$; b) zoom of the region near to the most negative Poisson's ratio (Point NP) of the CG-bounds: c) zoom of the region near to the Walpole Point (Point WP).

attain elastic properties close to the bounds in the region with high shear and low bulk moduli, near the points with negative Poisson $\AA \dot{Z}$ ratios denoted NP in Figure 7-a and b;

3) There are no substantial differences by using plane groups with high or low symmetries for capturing extreme materials close to the other two vertices defined by the CG-bounds.

\subsubsection{Problem target: Walpole Point (Problem 9 in Figure 7-a)}

The material layouts obtained with different plane groups for the WP point (of Figure 7-a and c) are depicted in Figure 8. These solutions confirm the conclusions reported by Sigmund [3]. The most extreme properties are attained with the symmetry $p 6 \mathrm{~mm}$ and the topology agrees with that reported by Sigmund in the same paper. Notably in this case, the microstructure is constituted by laminated bars and rigid joints. Sigmund has proven that parameterizing this topology and performing an optimization of the geometry configuration through these parameters, the material almost reaches the Walpole Point. It is evident that the additional symmetry lines of the $p 6 \mathrm{~mm}$ plane group guide the algorithm to attain a more adequate limit condition. 


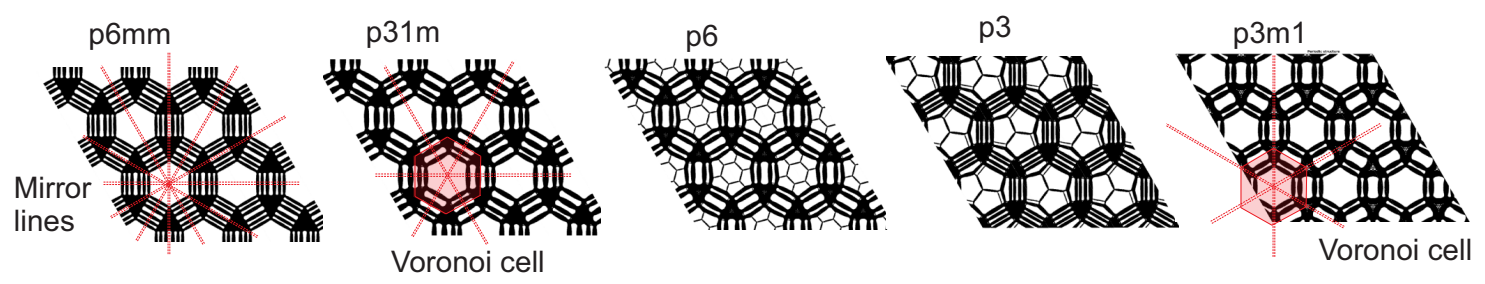

Figure 8: Two-dimensional design of extreme isotropic microstructures: Walpole Point WP in Figure 7 -a and c. Topologies obtained with plane groups $p 6 \mathrm{~mm}$, $p 31 m, p 6, p 3, p 3 m 1$. Mirror lines and Voronoi cells.

The same Figure 8 shows that when a lower symmetry is imposed, for example $p 3$, the algorithm searches for a solution tending to capture similar symmetry elements to those shown by the $p 6 \mathrm{~mm}$ plane group.

It is remarked that the Walpole point is asymptotically attained when one phase is void, both in two and three dimensions, such as mentioned in the introduction of the paper by Milton and Camar-Eddine [28].

\subsubsection{Problem target: minimum Poisson's ratio (Problem 2 in Figure 7-a)}

The resulting topologies for the problem identified with number 2 in Figure 7a, whose target is to attain the bound for the composite with the most negative Poisson's ratio (Point NP in the CG-bounds), are shown in Figure 9. In general, these topologies agree with the ones reported in the paper by Podestá et al. [15]. We note in Figure 7 -a and $b$ that the effective response is rather sensitive to the imposed plane group. The microarchitectures giving a good tendency are either chiral (obtained with the plane groups $p 6$ and $p 3$ without mirror symmetry lines) as well as not chiral (obtained with the plane group $p 31 \mathrm{~m}$ with one system of mirror symmetry line). Again, we note that $p 31 m, p 6$ and $p 3$ plane groups try to attain microstructures constituted by laminate bars. Note the similarity of the configuration attained with $p 6$ symmetry and the parameterized microstructure studied in Ostanin et al. [29].

The solutions with the plane groups $p 3 m 1$ and $p 6 m m$ are clearly inefficient if compared with the $p 6$ and $p 3$ solutions. This response can be explained by the mirror lines of the plane group $p 6 \mathrm{~mm}$ yielding an inadequate topology for emulating the deformation mechanisms typical of auxetic materials. The inefficiency of the solutions for both plane groups $p 3 \mathrm{~m} 1$ and $p 6 \mathrm{~mm}$ are additionally confirmed by observing the intricate resolved microarchitectures displayed in Figure 9.

The above discussed conclusions are valid for designing extreme composites whose target properties lay on a larger part of the left and upper CG-bounds, for example, for the problems denoted with the numbers 1 to 5 in Figure 7 -a. 


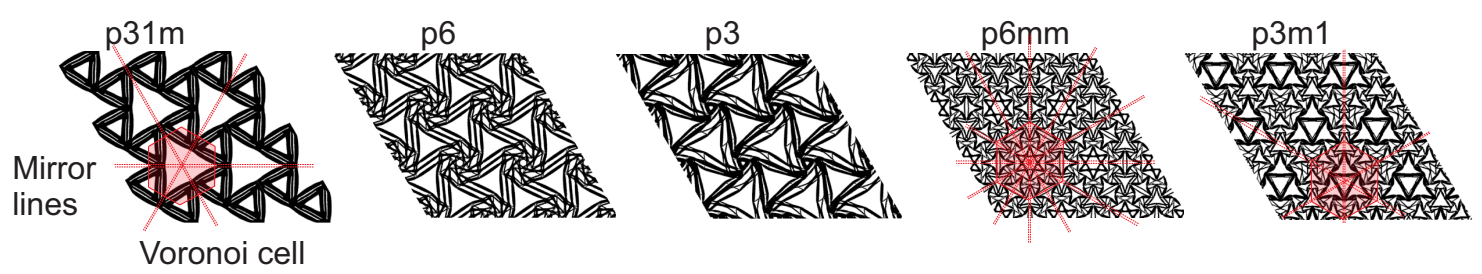

Figure 9: Two-dimensional design of isotropic microstructures: most negative Poisson's ratio (Point NP in Figure 7-a and b). Topologies obtained with plane groups $p 31 m, p 6, p 3, p 6 m m$, and $p 3 m 1$. Mirror lines and Voronoi cells.
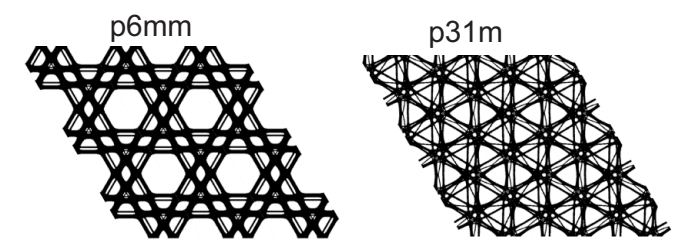

Figure 10: Two-dimensional design of extreme isotropic microstructures: maximum shear and bulk moduli (Problem 7 in Figure 7-a). Topologies obtained with plane groups $p 6 m m$ and $p 3 m 1$.

\subsubsection{Problem target: maximum shear and bulk moduli (Problem 7 in Figure 7-a)}

Similar microstructures are obtained with the five plane groups when searching for the stiffer microstructure at the upper right vertex of the CG-bounds, maximum shear and bulk moduli.

Figure 10 depicts the solutions attained with the plane groups $p 6 \mathrm{~mm}$ and $p 31 \mathrm{~m}$. They are similar microstructures to those reported by Sigmund [3].

\subsubsection{Smooth transition of topologies along the CG-bounds}

Figure 11 displays a sequence of the microarchitectures attained in problems 1 to 4 (with plane group $p 31 \mathrm{~m}$ ), as well as, 8 to 11 (with plane group $p 6 \mathrm{~mm}$ ). There can be seen a smooth and continuous transition of topologies for the problems along the CGboundary. These results open the possibility to define families of microarchitectures, via parametrization of their geometrical configuration, which could be used as a tool, by appealing to parameter optimization, for attaining topologies with properties even closer to the limits. 


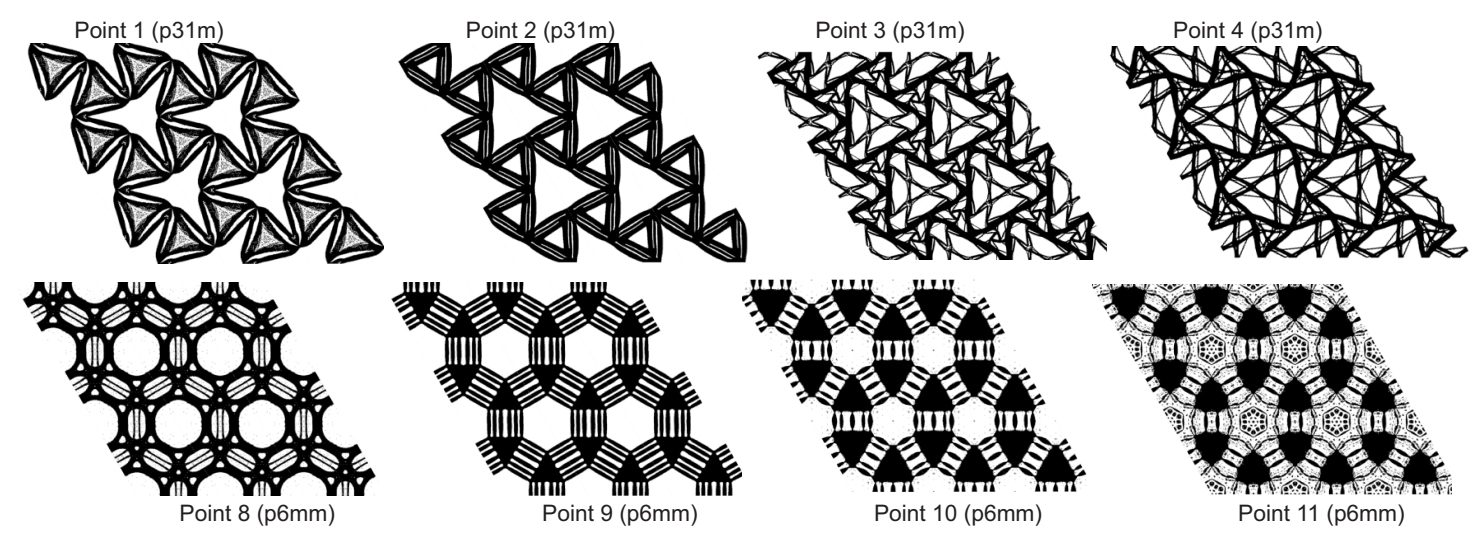

Figure 11: Transitions of topologies (Points 1 to 4 with $p 31 m$ plane group (above) and 8 to 11 with $p 6 \mathrm{~mm}$ plane group (below)). The problem numbers are identified in Figure 7-a.

\section{Design of 3-D isotropic materials}

Four different space groups $P 23, P m \overline{3} m$ (with SC Bravais lattices) and $I 23, \operatorname{Im} \overline{3} m$ (with BCC Bravais lattices) are adopted for designing the 3D topologies. All the solutions have been got with a Fast Fourier Transform procedure to compute the homogenized elastic properties. The cells have $100 \times 100 \times 100$ voxels in all cases.

Figure 12 plots the results in the space $(\hat{\kappa}, \hat{G})$. The HS-upper and lower bounds and some isolines of properties with negative Poisson's ratios are depicted, as well as 13 sets of solutions obtained with the four mentioned space groups. Each set of solutions are denoted with the numbers 1 to 13. Several instances have been run for every set of Problems 1 to 13 and for every space group. The solutions depicted in Figure 12 correspond to the best obtained case of all the runs for each space group.

\section{Isotropy analysis}

With the coefficients of the homogenized elasticity tensor $\hat{C}$, we compute the Zener ratios $^{13}$ of some representative microarchitectures as follows:

$$
\xi=\frac{\hat{C}_{44}}{\hat{C}_{11}-\hat{C}_{12}}
$$

A value 1 for this ratio indicates that $\hat{C}$ is isotropic ${ }^{14}$. Contrarily, a large value of $\xi$ indicates that $\hat{C}$ is far from being isotropic. Some Zener ratios are reported in

\footnotetext{
${ }^{13}$ The Zener ratio quantifies the anisotropy of the effective material properties having cubic symmetry.

${ }^{14}$ For the subsequent analysis, the elasticity tensors whose Zener ratios are different from 1 are projected to the space of isotropic tensors using the technique reported by Meille and Garboczi $[30]$.
} 


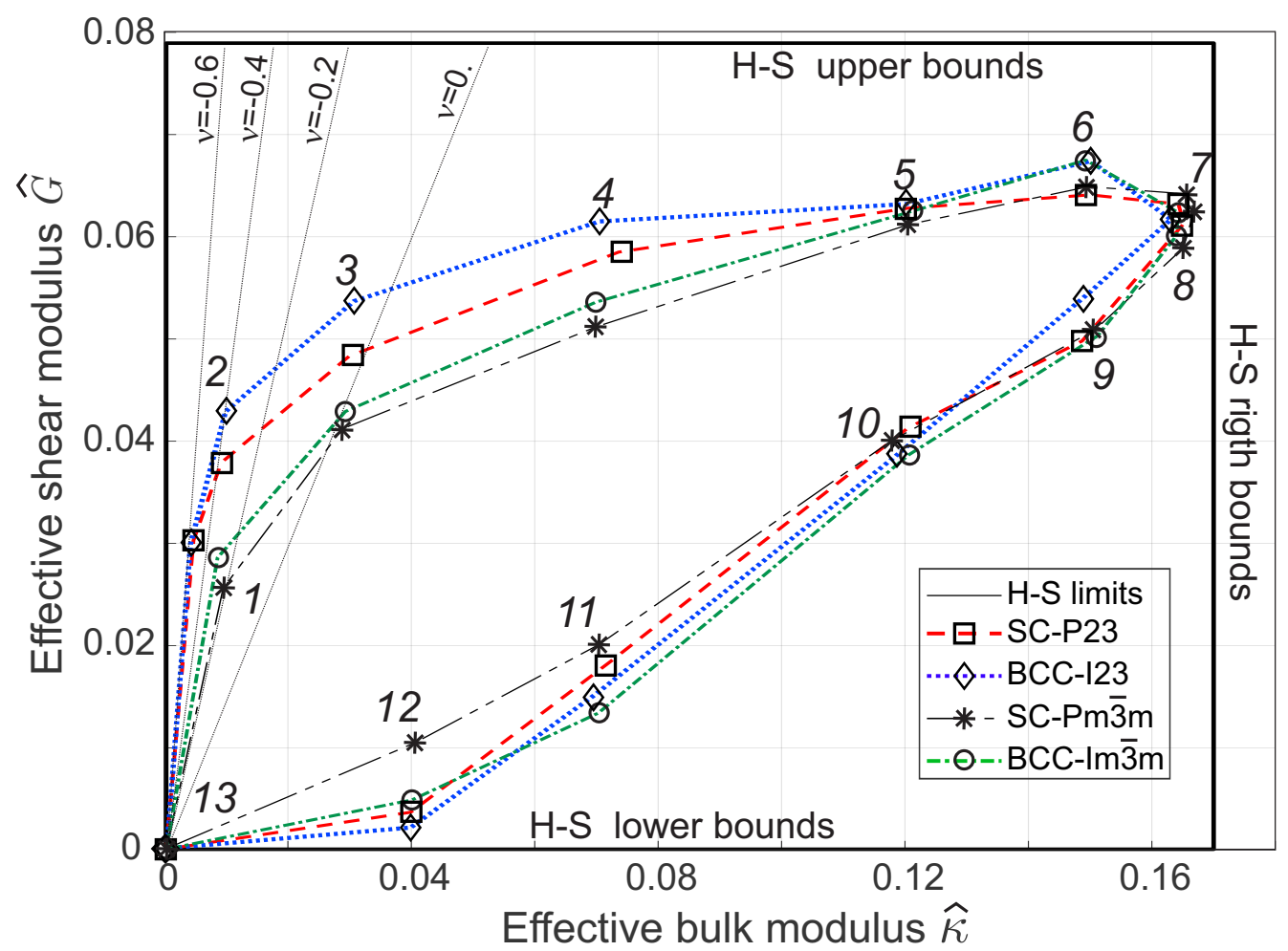

Figure 12: Three-Dimensional design of composites whose properties are close to the theoretical bounds. Hashin-Shtrikman bounds in the space $(\hat{\kappa}, \hat{G})$. Solutions for 13 different sets of problems using four space groups $P 23, \operatorname{Pm} \overline{3} m$ (with SC Bravais lattice) and $I 23, \operatorname{Im} \overline{3} m$ (with BCC Bravais lattice). Isolines of properties with negative Posson's ratios are shown. 
Table 3: Zener ratio $\xi$ quantifying the anisotropy of 3-D microarchitecture design solutions with space groups $P 23, I 23, P m \overline{3} m$ and $I m \overline{3} m$. The number of the problem in column 1 coincides with the problem number identified in Figure 12.

\begin{tabular}{|c|c|c|c|c|}
\hline Problem & $P 23$ & $I 23$ & $P m \overline{3} m$ & $I m \overline{3} m$ \\
\hline 1 & 1.009 & 1.012 & 0.943 & 0.995 \\
\hline 2 & 0.978 & 1.014 & 1.047 & 1.005 \\
\hline 7 & 1.035 & 1.010 & 1.004 & 0.987 \\
\hline 12 & 1.455 & 1.539 & 1.140 & 0.995 \\
\hline
\end{tabular}

Table 3. In general, they are close to 1, meaning that the effective response almost satisfies the isotropy constraint imposed on the topology optimization algorithm.

\subsection{Discussion of results}

After evaluating the results plotted in Figure 12 and Table 3, the following conclusions can be drawn:

1) In the region of the space $(\hat{\kappa}, \hat{G})$ corresponding to small $\hat{\kappa}$ and large $\hat{G}$, i.e., negative or small Poisson's ratios, the point group (either 23 or $m \overline{3} m$ ) have a higher influence than the Bravais lattice (SC or BCC) to achieve properties close to the bounds. The closest solutions are attained with the point group 23 , which has fewer symmetry elements than the $m \overline{3} m$ point group.

2) In the region of the space $(\hat{\kappa}, \hat{G})$ corresponding to high bulk and shear moduli, problems 6 and 7, the solutions are similar for identical lattices, independently of the point group. The reason for this response is that topologies with the highest symmetries display properties closer to the limit values in this specific region of the space $(\hat{\kappa}, \hat{G})$. Thus, when a space group with lower symmetry is imposed in the algorithm, it seeks for solutions with higher symmetry than the enforced one.

According to this conclusion, which is similar to that presented at the end of sub-Section 3.1.1, there would be a tendency to think that, in all cases, the imposition of the lowest symmetries is preferable to the highest ones and leaving to the algorithm, the search for higher symmetries, if it were the case. However, this conclusion is not correct in general. We have discussed in sub-Section 3.1.2 that the enforcement of appropriate symmetries has been beneficial for obtaining a marginally closer solution to the theoretical limits, as well as, to achieve notably more stable, robust and repetitive responses of the topology optimization algorithm. 
3) The following tendency has been observed in general for attaining the points on the HS-upper and right bounds. The BCC lattice provides solutions with larger values of $\hat{G}$, while the SC lattice provides solutions with slightly larger values of $\hat{\kappa}$.

4) In the HS-lower bound region, problem 12, where the HS-bound estimates the occurrence of pentamode materials, the solutions with space groups P23, I23 and $\operatorname{Im} \overline{3} m$ give lower shear stiffnesses. However, according to the results in Table 3, the P23 and I23 solutions cannot be considered as isotropic. Alternatively, the $\operatorname{Im} \overline{3} m$ solution attains a low shear modulus with a closely isotropic response.

\subsubsection{Microarchitecture transition along the upper bound}

An almost continuous transition of topologies with the imposed space group I23 is attained when designing microstructures with maximum shear modulus close to the HS-upper bound. These microarchitectures are depicted in Figure 13 and correspond to solutions of Problems 1, 2, 3, 4, and 6 of Figure 12. The attained microarchitectures are displayed from two opposite points of view, according to the directions [1 111$]$ and [ $\overline{1} \overline{1} \overline{1}]$, respectively ${ }^{15}$.

Due to the characteristic symmetries of space group $\operatorname{Im} \overline{3} m$, the topologies with this space group display an identical appearance when viewed from both directions [1 11 ] and [ $\overline{1} \overline{1} \overline{1}]$ because in this point group $m \overline{3} m$, both directions belongs to the same family $\langle 111\rangle$. Such property is not satisfied, in general, by topologies with I23 space group, property which is confirmed in Figure 13. However, the similar appearance observed from both points of view is noted in the solution of Problem 6, Figure 13.

The above observation confirms once again the previously discussed concept about that the imposition of a low symmetry condition, such as the I23, does not inhibit the attainment of topologies with higher symmetries, copying that of the space group $\operatorname{Im} \overline{3} m$. This observation is additionally supported by the fact that solutions obtained with space groups $I 23$ and $I m \overline{3} m$ have almost the same elastic properties stated in Figure 12,

\footnotetext{
${ }^{15}$ We use Miller indices to identify crystallographic directions and planes. A family of planes is denoted between braces " $\{\ldots\}$ " and collect all the planes which are related through the symmetry operations of the point group. Therefore, two families of planes with identical Miller indices but different point groups may indicate different sets of planes. The same observation is done for the family of directions denoted between angle brackets " $(. .\rangle$.$" ".$
} 


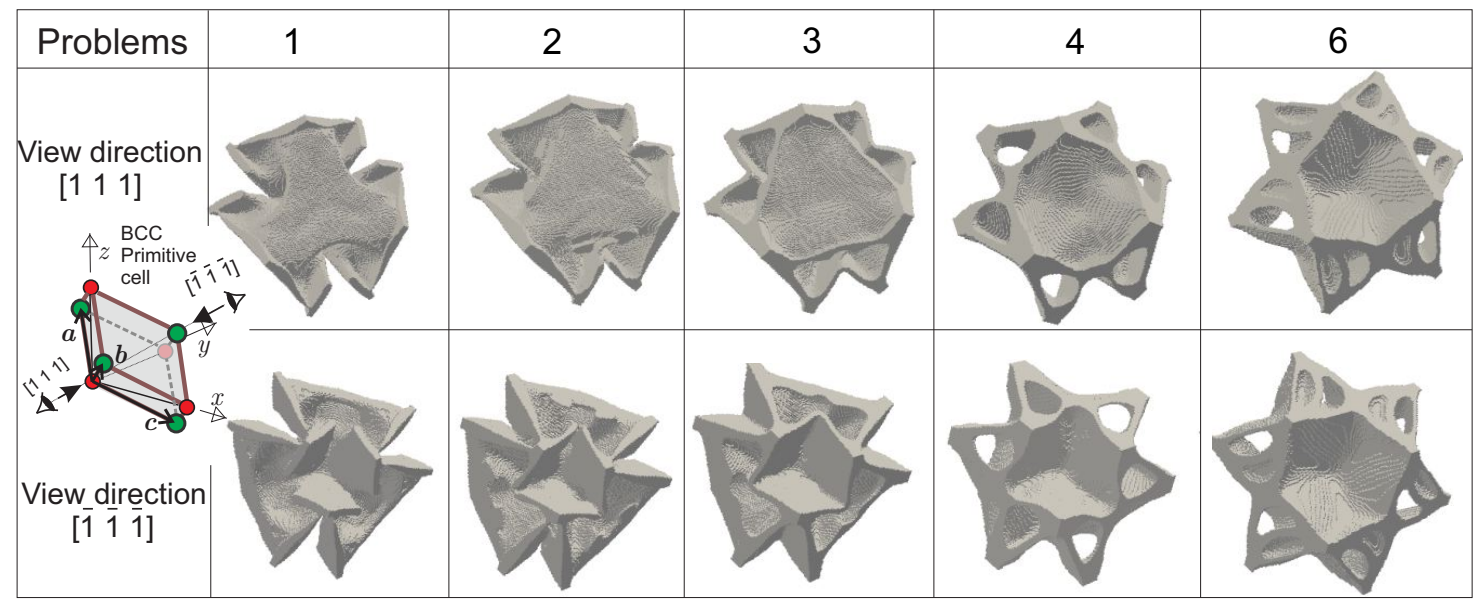

Figure 13: Microarchitecture transitions with maximal shear stiffness and target points along the HS-upper bound. Space group I23. Problems 1, 2, 3, 4 and 6 are in correspondence with those in Figure 12. Topologies of the BCC primitive cell observed from two points of view: [1 111$]$ and [ $\overline{1} \overline{1} \overline{1}]$.

\subsubsection{Microarchitecture of auxetic materials}

Several designed 3D microarchitectures with auxetic properties show topologies characterized by similar deformation mechanisms to those reported in the literature for $2 \mathrm{D}$ problems. A typical case is a microstructure designed to have minimum Poisson's ratio (Problem 1 in Figure 12) using the spatial group I23. Two different solutions are depicted in Figure 14 called Instances 1 and 2 . It can be noted that the conventional cell of Instance 1, Figure 14-c, consists of eight blocks, similar to rigid cubes, connected at their edges with joints. These rigid cubes are allowed to have a relative rotation between them. Thus, the deformation mechanism to get the required auxetic behavior is similar to the $2 \mathrm{D}$ microstructure reported in the literature and called rotating polygonal models, see Ren et al. [31] and Attard and Grima [32]. For comparison, the rotating polygonal microstructure is reproduced in Figure 14-e. Some other three-dimensional microarchitectures having a Poisson's ratio approaching -1 are reported in Milton [33].

One additional attained microstructure with similar elastic properties is denoted Instance 2. It is displayed in Figure $14 \mathrm{f}-\mathrm{i}$. The Voronoi cell of this microstructure shows four identical independent substructures which stay interconnected after the cell assembling. At the best of our knowledge, this microstructure has not been previously reported in the literature.

\section{Configurations resulting from the auxetic 3D microstructures projected onto cutting planes}

An additional aspect which shed some light on the understanding of the attained 


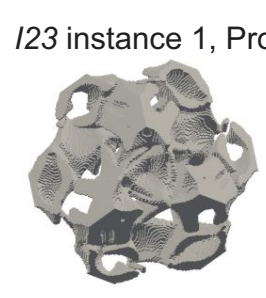

(a)

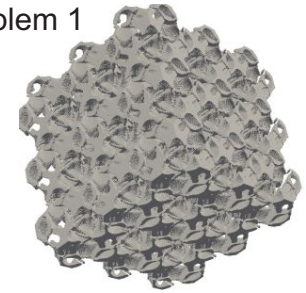

(b)

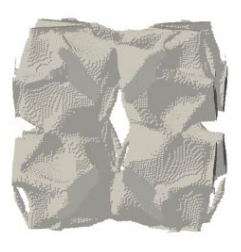

(c)

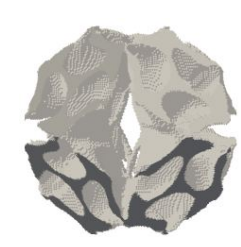

(d)

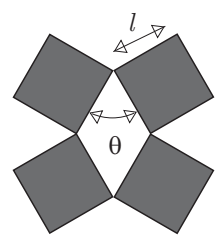

(e)

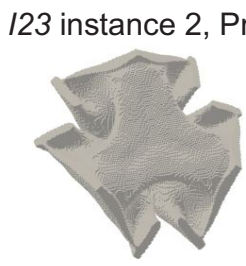

(f)

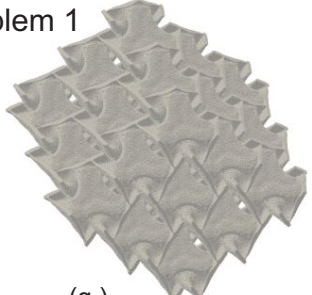

(g)

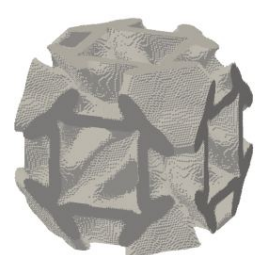

(h)

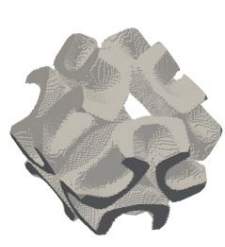

(i)

Figure 14: Two instances of composites with minimum Poisson's ratio attained with Problem 1 in Figure 12, space group I23. Instance 1: a) primitive cell; b) assembled microstructure; c) conventional cell; d) Voronoi cell; e) 2D rotating polygonal models described in Ren et al. [31]. Instance 2: f) primitive cell; g) assembled microstructure; h) conventional cell; i) Voronoi cell.

3D microstructures comes from the analysis of the resulting projected topologies by cutting the designed microarchitectures with specific families of planes. Of particular interest are those projected topologies onto planes containing symmetry elements.

We analyze the projected configuration onto planes orthogonal to three families of directions: $\langle 100\rangle,\langle 110\rangle$ and $\langle 111\rangle$.

This analysis is restricted to the solutions of problems 1 and 2 of Figure 12 displaying the most negative Poissson's ratios.

\section{a) Cutting planes orthogonal to $\langle 100\rangle$}

\section{a.1) Space groups $P 23$ and $I 23$}

The projected configurations onto generic planes orthogonal to $\langle 100\rangle$ of topologies with point groups 23 display a plane group $p 2$ with a square lattice. However, there are some specific positions of these cutting planes in where the projected topology may displays additional symmetry elements.

Particularly, when the position of the cutting plane is at the middle distance between two consecutive planes of the family $\{200\}$, the space group I23 projects as a plane group $p 2 g g$. This result is a consequence of the additional two-fold screw axes existing in the $I 23$ space group exhibited in Figure 5 -d. Therefore, the space group $P 23$ does not possess this symmetry. Figure 15, case A, display the projected configuration of both space groups onto this particular plane. Although 


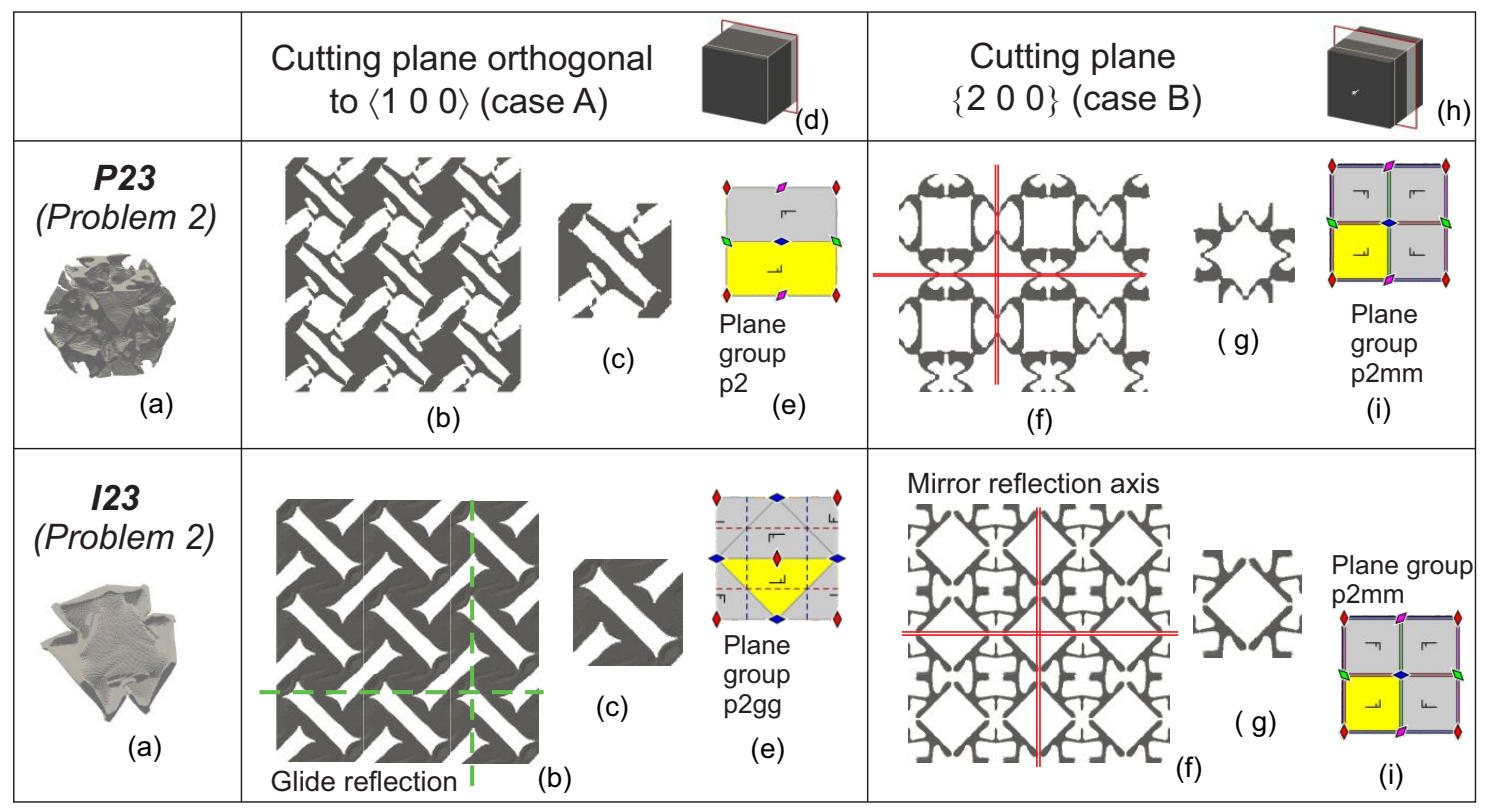

Figure 15: Projected microstructures attained of problem 2 in Figure 12. Space groups P23 and I23. Two projected configurations according to two different cut plane positions (cases A and B). a) primitive cells; b) and f) projected topologies of the assembled composites; c) and g) projected topologies of the conventional unit cells; d) positions in the conventional cell of the cutting planes; e) and i) plane groups and symmetry elements. The symbols representing the plane group symmetry elements are described in Figure 6. 


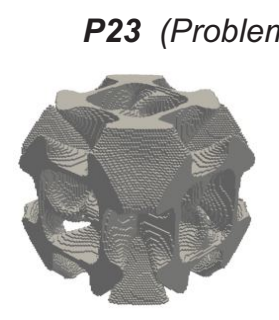

(a)

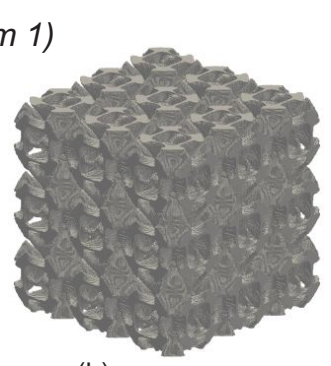

(b)

Cutting plane orthogonal to $\left\langle\begin{array}{lll}1 & 0 & 0\end{array}\right\rangle$

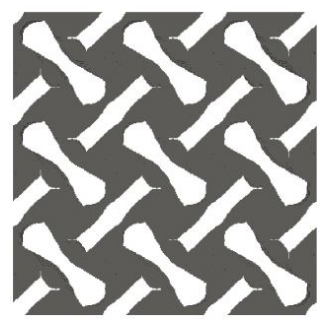

(c)

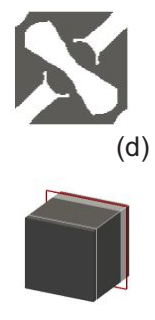

(e)

Figure 16: Microstructure attained with Problem 1 in Figure 12. Space group P23. a) primitive cell; b) assembled microstructure; c) projected topology of the assembled composite onto the plane orthogonal to $\langle 100\rangle$ situated between two consecutive planes of the family $\{200\}$; d) projected topology of the primitive unit cell onto the same plane; e) Position of the cutting plane relative to the primitive cell.

both configurations display analogous patterns, as a consequence of the additional glide reflection elements in the space group $I 23$, this microstructure presents a better resolution and, according to Figure 12, shows closer elastic properties to the bounds.

It is interesting to compare the similitude between the configuration displayed in Figure 15-b of space group I23, and the layout reported as antitetrachiral by Alderson et al. [34]. We observe that the here obtained projected topology is an anti-tetrachiral configuration. Notably, the anti-tetrachiral configuration is a $p 4 g m$ plane group. Such as mentioned by Alderson et al. [34], in 2D cases, the antitetrachiral configuration, if compared with alternative chiral configurations, is the most performer one for attaining Poisson's ratios close to -1, see Chen et al. [35].

Additionally, the topologies projected onto planes belonging to the family $\{200\}$, which contains two-fold rotation axes, have mirror lines resulting with a plane group $p 2 \mathrm{~mm}$, such as shown in Figure 15-f. This plane group penalizes the realization of auxetic materials. However, apparently, the arising of this detrimental configuration in a small number of planes is not sufficient to generate an inadequate response of the full microarchitecture.

Next, based on the results obtained in Problems 1 and 2 of Figure 12 with the spaces groups $P 23$ and $I 23$, we re-examine an issue already discussed above. We note that the imposition of a space group, $P 23$, with a less number of symmetry elements on the topology optimization algorithm, leaving it to attain the adequate configuration symmetry, does not work correctly in Problem 2. In this case, the solution with the I23 space group is notably better than the solution obtained with the P23 space group. However, this conjecture does work when analyzing the results of Problem 1. Examining the projected configuration of the P23 solution of Problem 1, depicted in Figure 16, we note that it tends to capture a higher symmetry, 


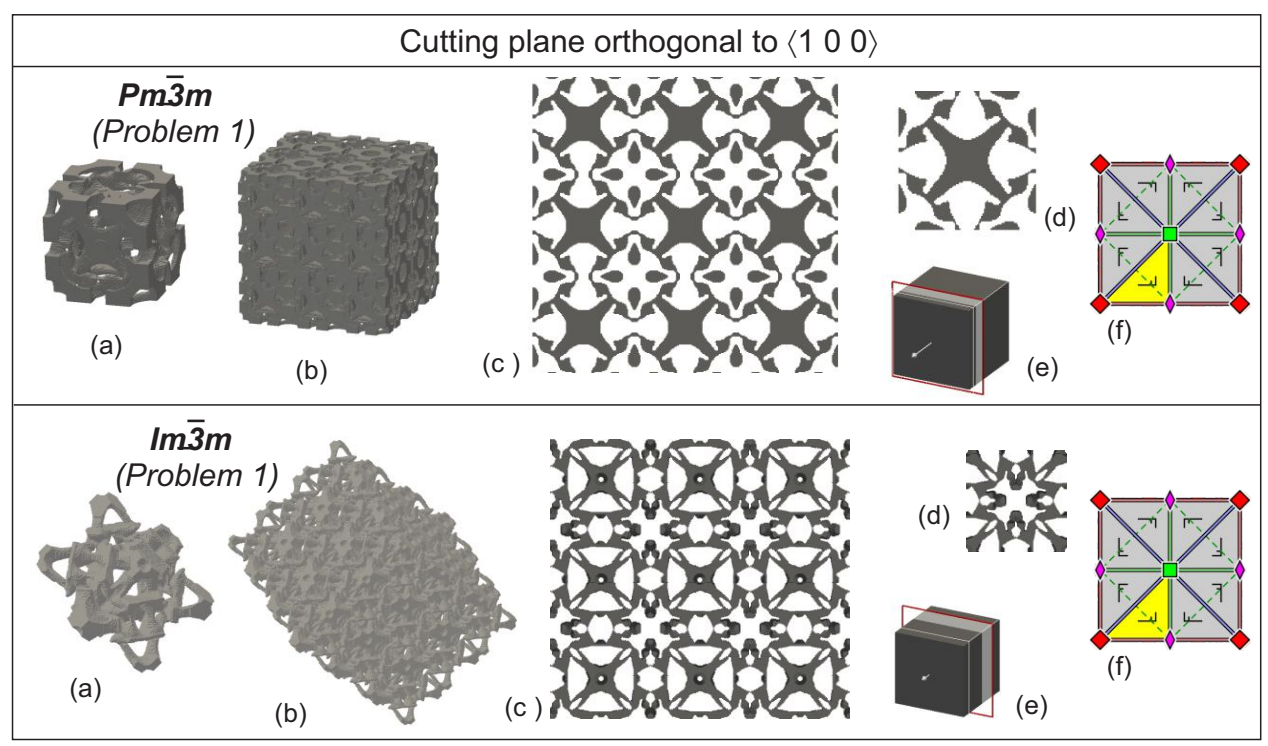

Figure 17: Microstructure attained with Problem 1 in Figure 12. Space groups $\operatorname{P} m \overline{3} m$ and $I m \overline{3} m$. a) primitive cells; b) assembled microstructures; c) projected topologies of the assembled composites onto a generic plane orthogonal to $\langle 100\rangle$; $d$ ) projected topologies of the conventional unit cells onto the same plane; e) Positions of the cutting planes relative to the conventional cells; f) symmetry elements of $\operatorname{P} m \overline{3} m$ and $\operatorname{Im} \overline{3} m$ configurations projected onto the generic plane. The symbols representing the plane group symmetry elements are described in Figure 6.

with mirror symmetry lines, and which additionally is similar to the topologies $I 23$ displayed in Figures 15-b.

At this point, it is necessary to distinguish between the point groups $p 2 m g$ and $p 2 \mathrm{~mm}$ and their capacity to achieve auxetics $2 \mathrm{D}$ composites. The glide symmetry element of the $p 2 \mathrm{mg}$ plane group is not necessarily so prejudicial as the mirror symmetry element is in the $p 2 \mathrm{~mm}$ case. There are several topologies in $2 \mathrm{D}$ having plane group $p 2 m g$ with isotropic elastic properties and negative Poisson's ratio, see the discussion in Section 5.1.5 of the paper by Podestá et al. [15].

\section{a.2) Space groups $P m \overline{3} m$ and $\operatorname{Im} \overline{3} m$}

The $P m \overline{3} m$ and $I m \overline{3} m$ space groups project onto a plane group $p 4 m m$ when the cutting plane is a generic plane orthogonal to $\langle 100\rangle$. Figure $17-f$ shows the symmetry elements of this plane group, and Figures 17-c and d display the material configurations of the designed microstructures of Problem 1 in Figure 12 projected onto this plane. Observe the complex topology shown by both microstructures.

According to the conclusions achieved for 2D topologies, the plane group $\mathrm{p} 4 \mathrm{~mm}$ involves a large number of mirror symmetry elements, which is very detrimental to 
obtain large negative Poisson's ratios. The same conclusion can be generalized to be applied to the results obtained in 3D designs with both spaces groups $P m \overline{3} m$ and $\operatorname{Im} \overline{3} m$. The bad performances displayed in Figure 12, Problems 1 and 2 , by the elastic properties of these space groups, can then be associated with this feature.

\section{b) Cutting planes orthogonal to $\langle 110\rangle$}

\section{b.1) Space groups $P 23$ and $I 23$}

The projected lattice onto the cutting planes orthogonal to $\langle 110\rangle$ generate a Rectangular Primitive lattice for the three-dimensional SC lattice and a Rectangular Centered one for the BCC lattice. In both cases, the aspect ratios of the conventional plane cells are $\sqrt{2}$.

The projections of the spaces groups $P 23$ and $I 23$ onto generic planes orthogonal to $\langle 110\rangle$ do not generate any symmetry element. Therefore, the plane group is $p 1$. However, in the particular case of the plane family $\{220\}$, which contains the twofold rotation axes, the plane group projected by the space groups $P 23$ and $I 23$ change to $\mathrm{pm}$ and $\mathrm{cm}$, respectively. Thus, the topologies obtained with $P 23$ and I23 for the Problem 2 projected onto that specific plane family are shown in Figure 18. Also, Figure 18-c identifies the symmetry lines of the projected configurations. Note that the plane group $\mathrm{pm}$ has two parallel symmetry mirror lines, while the $\mathrm{cm}$ has only one mirror line and one glide reflection line, such as exhibited in Figure 18-e. A consequence of these attributes is that the space group P23 displays a projected topology with two different types of triangles, while the $I 23$ displays only one type of triangle. It is interesting to compare the resemblance between these topologies with the 2D configuration depicted in Figure 9, p31m plane group, which is one of the best plane groups to attain negative Poisson's ratio.

\section{b.2) Plane groups $\operatorname{Pr} \overline{3} m$ and $\operatorname{Im} \overline{3} m$}

The space group $\operatorname{Pm} \overline{3} m$ projects onto the generic planes orthogonal to $\langle 110\rangle$ as a plane group $p 2 m m$ and the $I m \overline{3} m$ as a plane group $c 2 m m$. Again, and such as observed for $2 \mathrm{D}$ problems, for both space groups, the enforced symmetry onto these planes are not convenient for attaining negative Poisson's ratios.

\section{c) Cutting planes orthogonal to $\langle 111\rangle$}

\section{c.1) Plane groups $P 23$ and $I 23$}

The projected configurations onto generic planes orthogonal to $\langle 111\rangle$ of the space groups $P 23$ and $I 23$ display a configuration with a hexagonal Bravais lattice and plane group $p 3$. The symmetry elements of the plane group $p 3$ are shown in Figure 19-e. The topologies of the obtained microarchitectures with these space groups (P23 and I23) are displayed in Figure 19-b. These topologies have a tendency to attain a higher symmetry than the $p 3$ enforced by the space groups. 


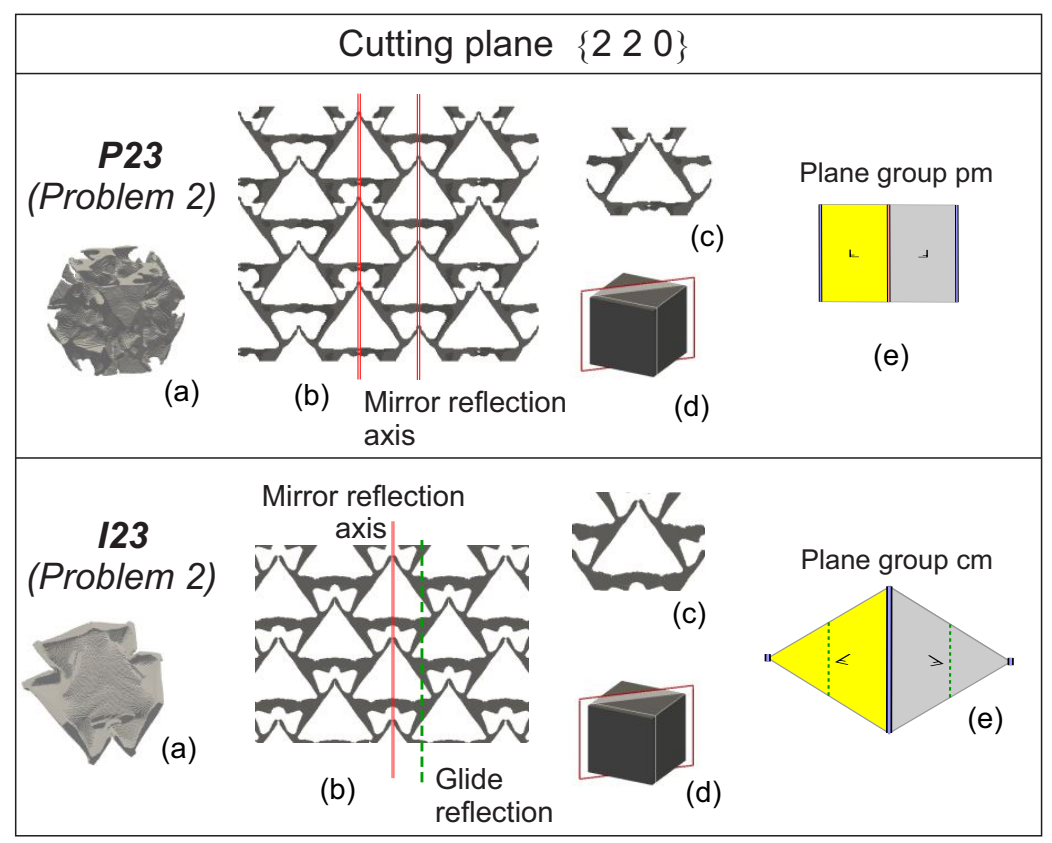

Figure 18: Projected microstructures attained with Problem 2 in Figure 12. Space groups P23 and I23. Cutting plane $\{220\}$. a) primitive cells; b) projected topologies of the assembled composites; c) projected topologies of the conventional unit cells; d) Positions of the cutting planes relative to the conventional cells; e) plane groups and symmetry elements. The symbols representing the plane group symmetry elements are described in Figure 6. 


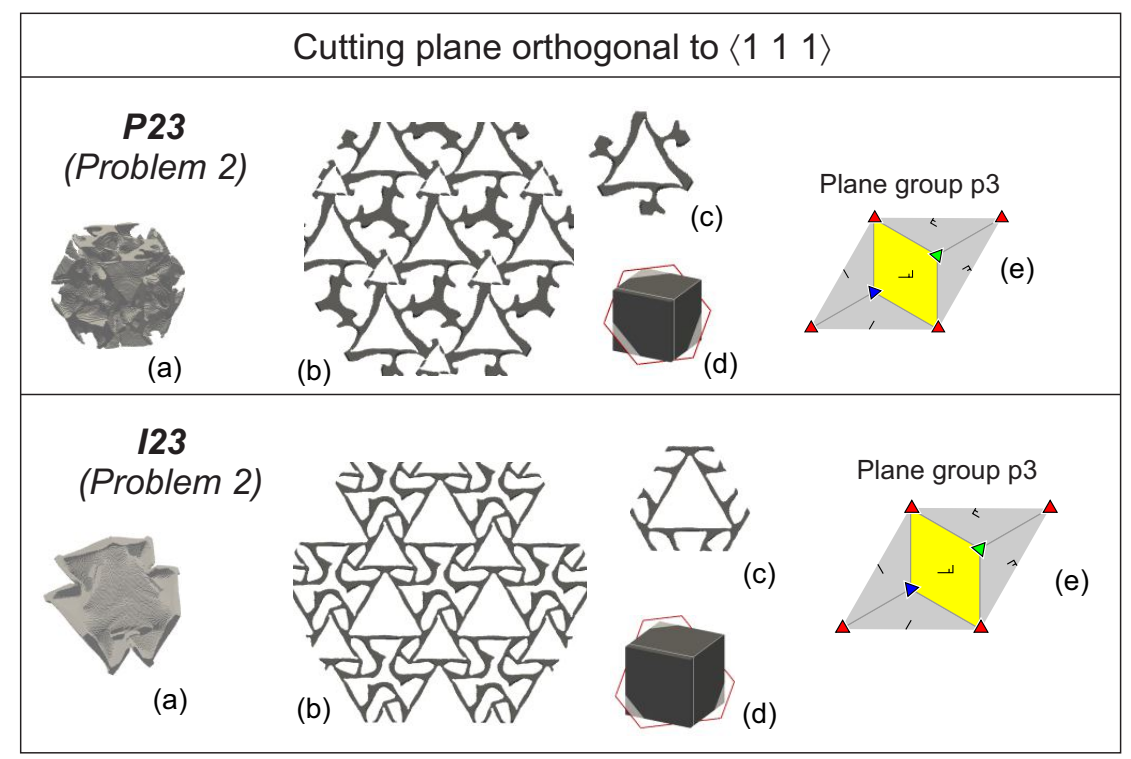

Figure 19: Projected microstructures attained with Problem 2 in Figure 19. Space groups P23 and I23. Column 1: Cutting planes orthogonal to $\langle 111\rangle$. a) primitive cells; b) projected topologies of the assemble composites; c) projected topologies of the conventional unit cells; d) Positions of the cutting planes relative to the conventional cells; e) plane groups and symmetry elements. The symbols representing the plane group symmetry elements are described in Figure 6. 


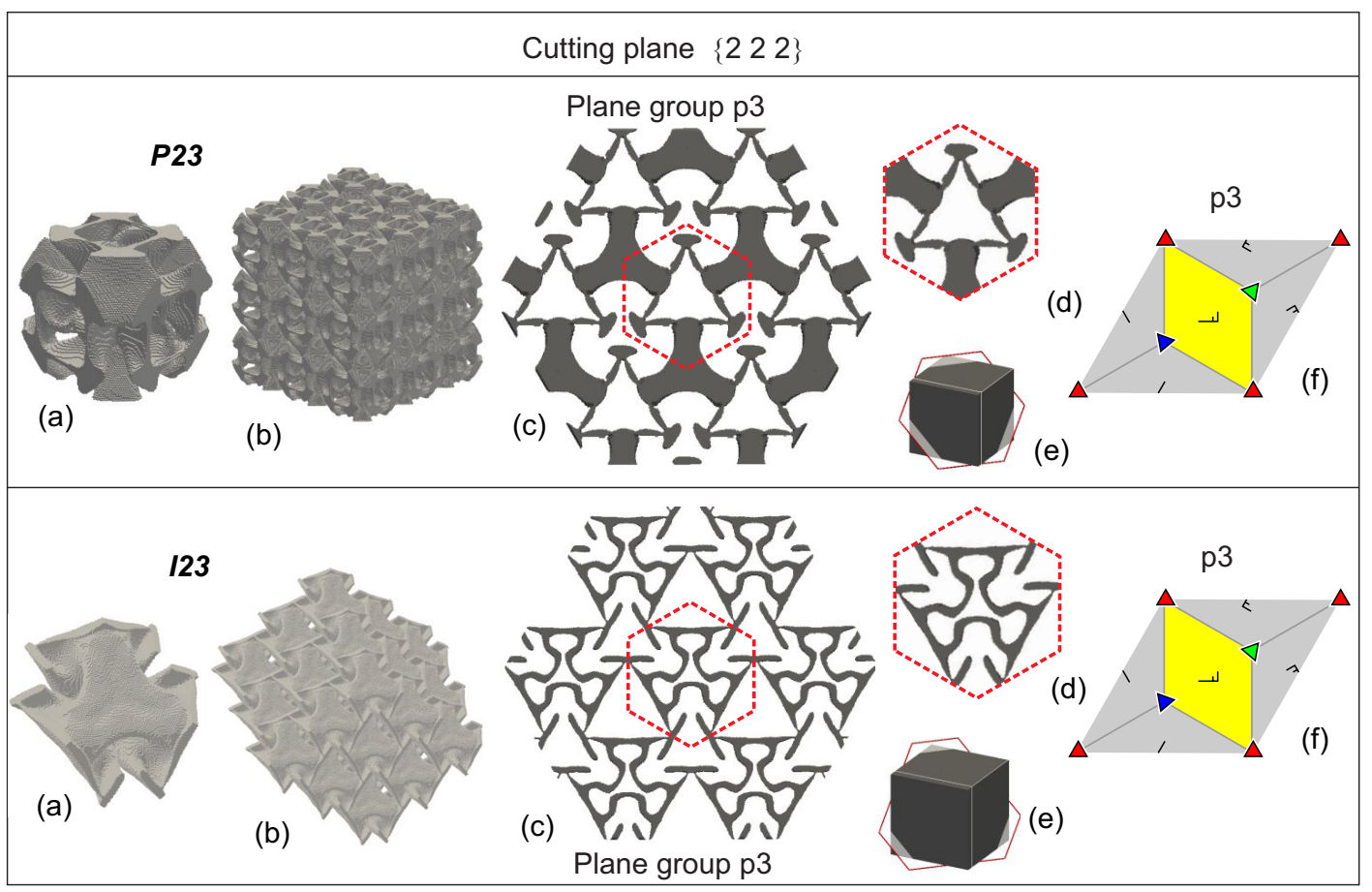

Figure 20: Microstructure attained with Problem 1 in Figure 12. Space groups P23 and I23. a) primitive cells; b) assembled microstructure; c) projected topologies of the assembled composite onto the family of planes $\{222\}$; d) Voronoi unit cell of the projected topologies; e) Positions of the cutting planes relative to the conventional cells; f) Projected symmetry elements onto a generic plane orthogonal to $\langle 111\rangle$ of space groups $P 23$ and I23. The symbols representing the plane group symmetry elements are described in Figure 6.

The topologies attained in Problem 1 of Figure 12 projected onto planes orthogonal to $\left\{\begin{array}{lll}1 & 1 & 1\end{array}\right\}$ almost copy, even with more confidence than in projections onto generic planes $\langle 111\rangle$, a plane group $p 3 m 1$. They are shown in Figure 20-c and d.It is notable that the algorithm searches for configurations with higher symmetries than the imposed ones also on planes orthogonal to $\langle 100\rangle$ and $\langle 110\rangle$.

Analyzing the attained symmetries in these cases, we observe that they are compatible with space group configurations $P \overline{4} 3 m$ and $I \overline{4} 3 m$ projected onto the same family of planes. This conclusion may be a symptom that these space groups are better than the imposed ones for reaching the problem target.

\section{c.2) Plane groups $\operatorname{Pr} \overline{3} m$ and $\operatorname{Im} \overline{3} m$}

Figure 21 shows the topologies attained with $P m \overline{3} m$ space group and projected onto cutting planes orthogonal to $\langle 111\rangle$. In this generic plane, the projected topology has $p 3 m 1$ plane group. Particularly, if the cutting plane belongs to the 


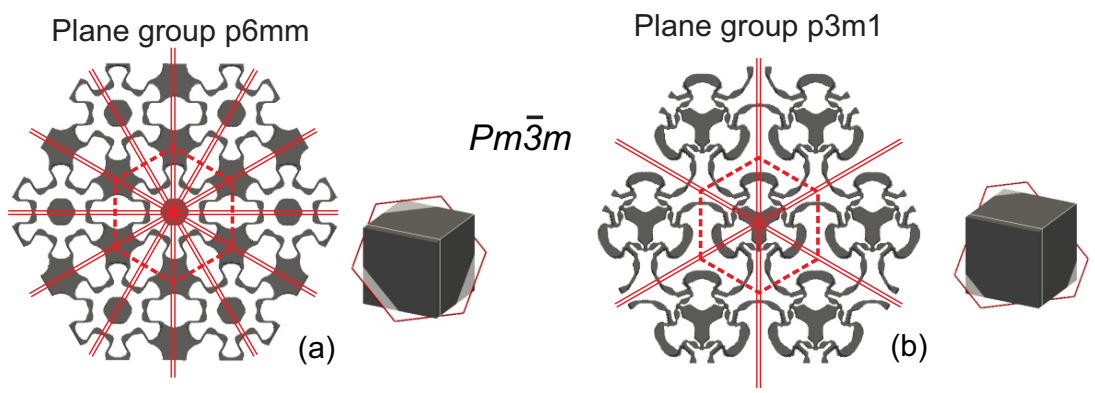

Figure 21: Projected topologies onto planes orthogonal to $\langle 111\rangle$ of the microarchitectures at Problem 1 in Figure 12, space group $P m \overline{3} m$. a) cutting plane belongs to the family $\{222\}$; b) generic cutting plane orthogonal to $\langle 111\rangle$.

family $\{222\}$, which contains the inversion center of the point group element $\overline{3}$, the plane group is a $p 6 \mathrm{~mm}$. Identical symmetry properties are valid for topologies with $\operatorname{Im} \overline{3} m$ space groups, except that the family of planes containing the inversion centers is the $\{444\}$.

Again in this case, the results obtained with the space group $P m \overline{3} m$ and $\operatorname{Im} \overline{3} m$ confirm the comment mentioned in sub-Section 3.1.2 about that the plane group p6mm penalizes severely the capturing of auxetic materials.

\subsubsection{Microarchitectures for pentamode materials}

The lower estimation of the HS-bounds corresponds to isotropic materials with close to zero shear modulus. Therefore, according to the denomination of Milton and Cherkaev [2], they are pentamode materials ${ }^{16}$.

By solving the isotropic problem of minimum shear modulus for the bulk modulus $\hat{\kappa}=0.04$ (Problem 12 in Figure 12), we obtain a similar microstructure to that reported in the literature for this family of metamaterials, see Milton and Cherkaev [2], Kadic et al. [37] and Kadic et al. [38]. As can be observed in Figure 22, the microstructures obtained using the present methodology with space groups P23 and Im $\overline{3} m$ are constituted by two independent sub-microarchitectures in the cubic cell, respectively. Each sub-microstructure is approximately similar to the ones reported by Kadic et al. [37] in Figure 1-b and are, typically, constituted by rigid elements connected through flexible joints.

\footnotetext{
${ }^{16} \mathrm{~A}$ Pentamode material is a class of extremal material having five easy (compliant) modes of deformation in a three-dimensional space, and having only one non-easy (stiff) mode of deformation. The elasticity tensor of this material has one non-null eigenvalue and five null eigenvalues (hence the name of pentamode given to this class of materials). In 1995, Milton and Cherkaev [2] have coined the name of pentamode materials in the context of linear elasticity. In the same year, Sigmund [36] has independently introduced it in the context of inverse homogenization problems.
} 


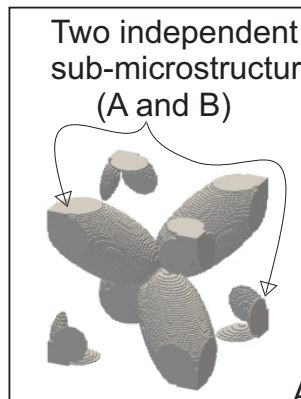

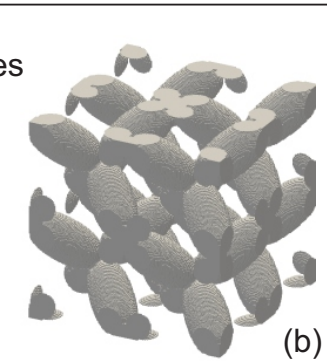

Assembled microstructures

(a)

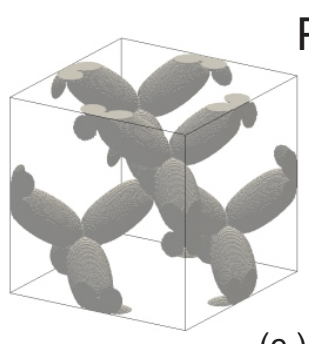

(c)
P23

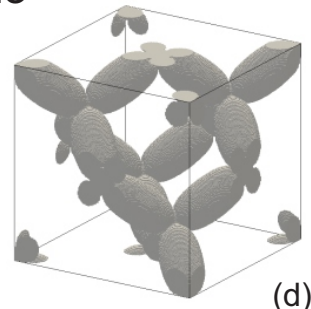

(d)

Sub-microstructure A Sub-microstructure B

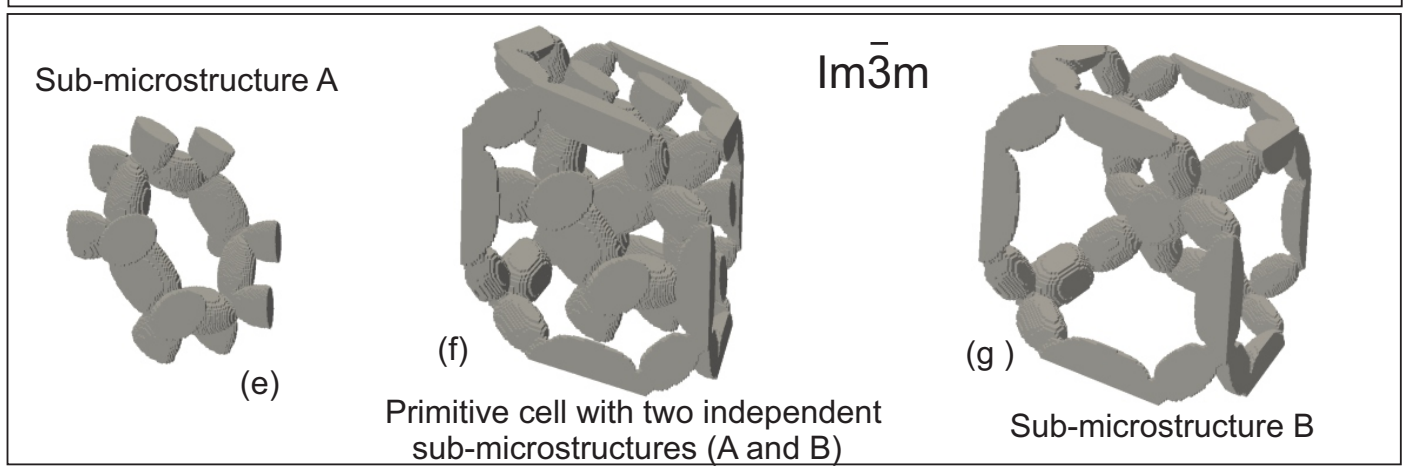

Figure 22: Topologies attained with Problem 12 in Figure 12 by enforcing a space group $P 23$ (a to d) and $\operatorname{Im} \hat{3} m$ (e to g). a) and f) are the primitive unit cells; b) assembled microstructures; c, d e and g) sub-microstructures. 
The homogenized elasticity tensors of the microstructure obtained with the P23 space group has the following six eigenvalues: $0.1196 ; 0.0098 ; 0.0098 ; 0.0084 ; 0.0084$ ; 0.0084, and the ratio between the maximum and minimum is approximately 14 .

The homogenized elasticity tensors of the microstructure obtained with the $\operatorname{Im} \overline{3} m$ space group has the following six eigenvalues: $0.1204,0.0098 ; 0.0098 ; 0.0098$; 0.0098; 0.0098. The ratio between the maximum and minimum is approximately 12 .

The I23 solution, which gives the lowest shear modulus, display eight submicrostructures, and a second instance of the $P 23$ solution, not shown here, displays four sub-microstructures. Thus, according to the present results, we perceive that an increase in the number of sub-microstructures entails a decrease of the shear modulus.

\section{Conclusions}

In this paper, we have analyzed the role that the symmetries of crystals play in the topology design of isotropic elastic materials when the design target is to attain properties close to the theoretical bounds, and the optimum design methodology is based on an inverse homogenization technique. The summary of the attained results are depicted in Figures 7 and 12. They are a demonstration that the maximal/minimal achievable properties strongly depend on the crystal symmetries imposed on the mathematical technique. And most important, the adequate crystal symmetries to reach these maximal/minimal properties change with the limit target point, or sector, on the CG and HS-bounds in the plane $(\hat{\kappa}, \hat{G})$. In both cases, $2 \mathrm{D}$ and $3 \mathrm{D}$, we have found that the bounds on the right part of the plane, with maximum $\hat{\kappa}$, can be approximated with the higher crystal symmetries. Contrarily, to approach the left bound, it is better to explore other types of symmetries.

In $2 \mathrm{D}$ problems, the isotropy of the composite is guaranteed by adopting plane group symmetries consistent with the hexagonal crystal system. However, not all these plane groups have identical effects. For example, to attain auxetic composites, it is convenient to explore the $p 3, p 31 \mathrm{~m}$ and $p 6$ plane groups, while the $p 6 \mathrm{~mm}$ and $p 3 m 1$ are unsuitable in these cases. Notably, the plane groups $p 6$ and $p 3$ allow for the development of chiral configurations. Conclusions are different if the extreme target properties are the maximal bulk and minimal shear moduli (the Walpole Point). In this case, the plane group $p 6 \mathrm{~mm}$ is the most effective one. For this particular problem, we have also shown that the imposition of a plane group with low symmetry, such as $p 3$, does not provide as good solutions as those attained by imposing the $p 6 \mathrm{~mm}$ plane group. This result proves that a procedure which takes a plane group with low symmetry $(p 3)$ and leaving the algorithm for searching the higher symmetry $(p 6 \mathrm{~mm})$ does not work, in general, with the best performance.

In $3 \mathrm{D}$ problems, we have only analyzed symmetries of the cubic crystal system. 
In this case, the dependence on the space group for attaining maximal $/ \mathrm{minimal}$ properties is still more pronounced than in 2D cases. The space groups I23 and P23 are notably more suitable to attain maximal properties in the region close to the HS-left-upper bound, coincident with the response of auxetic materials. A similar conclusion about the proper selection of the cell defining the optimization domain $\Omega_{\mu}$ can be drawn. The BCC primitive cell has turned out to be notably more advantageous than the simple cubic (SC) cell to get maximal properties in some sectors of the plane $(\hat{\kappa}, \hat{G})$, along the HS-upper bound.

Even when we have not specifically analyzed the numerical response of the topology optimization algorithm, we envisage that the additional crystal symmetry constraints notably increase the robustness and stability of the algorithm, by limiting the search space of the geometrical variables defining the topology.

Finally, we remark that the use of crystal symmetries for topology design can be easily extended to other more general thermal, photonic, acoustic microarchitecture design problems, anticipating similar potential benefits to those here explored.

\section{Acknowledgments}

The authors acknowledge the financial support from CONICET and ANPCyT (grants PICT 2014-3372 and 2016-2673).

\section{References}

[1] M. Kadic, G.W. Milton, M. van Hecke, and M. Wegener. 3D metamaterials. Nature Reviews Physics, 1:198-210, 2019.

[2] G.W. Milton and A.V. Cherkaev. Which elasticity tensors are realizable? Journal of Engineering Materials and Technology, 117(4):483-493, 1995.

[3] O. Sigmund. A new class of extremal composites. Journal of the Mechanics and Physics of Solids, 48(2):397-428, 2000.

[4] E. Prince; Boston ; London, editor. International Tables for Crystallography. Volume C, Mathematical, Physical and Chemical Tables. Dordrecht, 2004. Published for the International Union of Crystallography by Kluwer Academic Publishers.

[5] B.K. Vainshtein. Fundamentals of crystals: Symmetry, and methods of structural crystallography, volume 1. Springer Science \& Business Media, 2013.

[6] J. Sólyom. Fundamentals of the Physics of Solids: Volume 1: Structure and Dynamics. Springer Science \& Business Media, 2007. 
[7] J.F. Nye. Physical Properties of Crystals: Their Representation by Tensors and Matrices, volume 146. Clarendon Press-Oxford., 2006.

[8] O. Sigmund. Materials with prescribed constitutive parameters: an inverse homogenization problem. International Journal of Solids and Structures, 31 (17):2313-2329, 1994.

[9] S. Torquato. Optimal design of heterogeneous materials. Annual Review of Materials Rresearch, 40:101-129, 2010.

[10] M. Osanov and J.K. Guest. Topology optimization for architected materials design. Annual Review of Materials Science, 46:211-233, 2016.

[11] GW Milton. Stiff competition. Nature, 564(7734):E1, 2018.

[12] M.P. Bendsoe and O. Sigmund. Topology Optimization: Theory, Methods, and Applications. Springer Science \& Business Media, 2003.

[13] E. Andreassen and O. Lazarov, B.S.and Sigmund. Design of manufacturable 3D extremal elastic microstructure. Mechanics of Materials, 69(1):1-10, 2014.

[14] J.M. Podestá, C. Méndez, S. Toro, A.E. Huespe, and J. Oliver. Material design of elastic structures using voronoi cells. International Journal for Numerical Methods in Engineering, 115(3):269-292, 2018.

[15] J.M. Podestá, C.G. Méndez, S. Toro, and A.E. Huespe. Symmetry considerations for topology design in the elastic inverse homogenization problem. Journal of the Mechanics and Physics of Solids, 128:54-78, 2019.

[16] C.G. Méndez, J.M. Podestá, S. Toro, A.E. Huespe, and J. Oliver. Making use of symmetries in the three-dimensional elastic inverse homogenization problem. Journal for Multiscale Computational Engineering, 17:261-280, 2019.

[17] R. Yera, N. Rossi, C. Méndez, and A.E. Huespe. Three-dimensional material microstructures dataset displaying extreme isotropic elastic properties. 2019. Mendeley Data, v1 http://dx.doi.org/10.17632/cc2hgr9kvh.2.

[18] A.V. Cherkaev and L.V. Gibiansky. Coupled estimates for the bulk and shear moduli of a two-dimensional isotropic elastic composite. Journal of the Mechanics and Physics of Solids, 41(5):937-980, 1993.

[19] Z. Hashin and S. Shtrikman. A variational approach to the theory of the elastic behaviour of multiphase materials. Journal of the Mechanics and Physics of Solids, 11(2):127-140, 1963. 
[20] M.F. Thorpe and I. Jasiuk. New results in the theory of elasticity for twodimensional composites. Proceedings of the Royal Society of London. Series A: Mathematical and Physical Sciences, 438(1904):531-544, 1992.

[21] J.G. Berryman and G.W. Milton. Microgeometry of random composites and porous media. Journal of Physics D: Applied Physics, 21(1):87, 1988.

[22] S. Amstutz and H. Andrä. A new algorithm for topology optimization using a level-set method. Journal of Computational Physics, 216(2):573-588, 2006.

[23] S. Amstutz, S.M. Giusti, A.A. Novotny, and E.A. de Souza Neto. Topological derivative for multi-scale linear elasticity models applied to the synthesis of microstructures. International Journal for Numerical Methods in Engineering, 84(6):733-756, 2010.

[24] A.A. Novotny and J. Sokołowski. Topological derivatives in shape optimization. Springer Science \& Business Media, 2012.

[25] E. Hitzer and C. Perwass. Visualization of fundamental symmetries in nature. In Proceedings of Fuzzy System Symposium (FSS 2009), Tsukuba, Japan, 14-16 Jul., 2009. http://spacegroup.info/.

[26] D.J. Eyre and G.W. Milton. A fast numerical scheme for computing the response of composites using grid refinement. The European Physical JournalApplied Physics, 6(1):41-47, 1999.

[27] N. Rossi Cabral, R. Yera, C.G. Méndez, and A.E. Huespe. Numerical technique for the $3 \mathrm{~d}$ microarchitecture topology design of elastic composites inspired on crystal symmetries. submitted to: Computer methods in applied mechanics and engineering, 2019.

[28] G.W. Milton and M. Camar-Eddine. Near optimal pentamodes as a tool for guiding stress while minimizing compliance in 3d-printed materials: A complete solution to the weak g-closure problem for 3d-printed materials. Journal of the Mechanics and Physics of Solids, 114:194-208, 2018.

[29] I. Ostanin, G. Ovchinnikov, D.C. Tozoni, and D. Zorin. A parametric class of composites with a large achievable range of effective elastic properties. Journal of the Mechanics and Physics of Solids, 118:204-217, 2018.

[30] S. Meille and E.J. Garboczi. Linear elastic properties of 2d and 3d models of porous materials made from elongated objects. Modelling and Simulation in Materials Science and Engineering, 9(5):371, 2001. 
[31] X. Ren, R. Das, P. Tran, T.D. Ngo, and Y.M. Xie. Auxetic metamaterials and structures: A review. Smart Materials and Structures, 2018.

[32] D. Attard and J.N. Grima. A three-dimensional rotating rigid units network exhibiting negative poisson's ratios. Physica Status Solidi (b), 249(7):13301338, 2012.

[33] G.W. Milton. New examples of three-dimensional dilational materials. physica status solidi (b), 252(7):1426-1430, 2015.

[34] A. Alderson, K.L. Alderson, D. Attard, K.E. Evans, R. Gatt, J.N. Grima, W. Miller, N. Ravirala, C.W. Smith, and K. Zied. Elastic constants of 3-, 4and 6-connected chiral and anti-chiral honeycombs subject to uniaxial in-plane loading. Composites Science and Technology, 70(7):1042-1048, 2010.

[35] Y.J. Chen, F. Scarpa, Y.J Liu, and J.S. Leng. Elasticity of anti-tetrachiral anisotropic lattices. International Journal of Solids and Structures, 50(6):9961004, 2013.

[36] O. Sigmund. Tailoring materials with prescribed elastic properties. Mechanics of Materials, 20(4):351-368, 1995.

[37] M. Kadic, T. Bückmann, N. Stenger, M.l Thiel, and M. Wegener. On the practicability of pentamode mechanical metamaterials. Applied Physics Letters, 100(19):191901, 2012.

[38] M. Kadic, T. Bückmann, R. Schittny, P. Gumbsch, and M. Wegener. Pentamode metamaterials with independently tailored bulk modulus and mass density. Physical Review Applied, 2(5):054007, 2014. 